\title{
Micromanaging time: Measuring and controlling timing errors in computer-controlled experiments
}

\author{
CARL P. CREEGER, KEVIN F. MILLER, and DAVID R. PAREDES \\ University of Texas, Austin, Texas
}

\begin{abstract}
Computer-controlled experiments present a number of potential sources of error in timing the presentation of events, including video refresh rate, keyboard scanning rate, and disk I/O times. A terminate-and-stay-resident routine implementing multiple millisecond-accuracy timers is presented. Interfaces permitting use of the timer with several higher level languages (C, FORTRAN, Pascal, and QuickBASIC) are described, as is a design for a two- to four-button response system using the computer's printer port. A general strategy is described for using multiple timers to control and measure variation in critical experimental events. A C language program is provided to benchmark variation in the time required to perform common experimental tasks (screen refreshing, switching video pages, disk $\mathrm{I} / 0$, loop calculations), and results are summarized for several representative computers that use the IBM design.
\end{abstract}

Since at least the time of Donders (1868/1969), a standard psychological procedure has involved presenting subjects with visual stimuli and measuring the time they take to produce some response. The advent of microcomputers has greatly facilitated studies based on this general paradigm, but it has also introduced a number of potential errors in timing the events that are under experimental control.

The term micromanagement (LeMay, Lerner, \& Taylor, 1988) was coined in the 1970s (Barnhart, 1984) to refer to the detailed planning of local operations by a central executive, a practice that can have the consequence of leaving little room for local initiative. Using personal computers to obtain precise timing of events requires a sort of micromanagement, in which the timing of events is not affected by ongoing computational processes. The present paper describes: (1) sources of timing errors in computer-controlled experiments, (2) an implementation of millisecond-level timing that is both flexible and modular, (3) a strategy for using the timing routine to measure and minimize timing errors, and (4) a benchmark program for comparing the performance of experimentally relevant tasks on different IBM PC computers.

\section{Sources of Timing Errors}

Where timing errors are constant across trials, they may be of little experimental significance, although variations in stimulus onset asynchrony (SOA) in priming experi-

The software development reported here was supported by NSF Grant BNS-8510546 and grants of equipment from the IBM Corporation to the second author through Project Quest. We would like to acknowledge helpful comments on an earlier version of this paper by Nancy Lobaugh and Colin MacLeod. Address reprint requests to: Kevin F. Miller, Department of Psychology, Mezes 330, University of Texas, Austin, TX 78712 . ments of as little as $100 \mathrm{msec}$ can be of theoretical importance (Posner, 1978).

Time to generate stimuli. The largest timing errors result from ignoring the time required to generate complex experimental stimuli. Preparing a visual display on an IBM AT can easily take several hundred milliseconds. This error is likely to be constant across trials, but it is large enough to affect the meaning of SOAs.

Video refresh rate. Computer displays are changed by a cyclical process in which each horizontal line is updated in turn. The mechanics of screen updating pose problems for presenting a new stimulus at a predetermined time $(\mathrm{Pa}-$ redes, Miller, \& Creeger, 1990). In particular, failure to start timing when the stimulus is actually presented (immediately following vertical retraces) can result in maximum timing errors equal to the screen refresh interval (16.7 msec on an EGA display). One solution to the problem of synchronizing stimulus presentation to screen refreshes is to draw the stimulus during the interval (about $1.7 \mathrm{msec}$ on an EGA) when vertical retracing occurs (Dlhopolsky, 1989). For stimuli too complicated to be created in $1.7 \mathrm{msec}$, this problem can be minimized by writing stimulus information to an invisible graphics "page" and then making that page visible in synchrony with the start of the next screen refresh cycle. The PSYBENCH program takes advantage of this technique, which is described in detail in Paredes, Miller, and Creeger (1990).

Disk $\mathbf{1} / \mathbf{O}$. The time required to read and write information to a hard disk depends on the arrangement of data on the disk. The operating system (MS-DOS) assigns data to physical disk locations; it frequently breaks up files and writes sectors wherever space is available. As the results of the benchmark testing reported below indicate, disk I/O results in the largest source of variable error of those described in this section. Careful program design usually 
allows disk-intensive operations to be performed during portions of the experiment that are not time-critical.

Keyboard and button I/O. IBM PCs and compatibles use a scanned matrix keyboard with a serial data connection from the keyboard to the computer. Scanning the entire keyboard takes $8 \mathrm{msec}$, which results in a variable error of $\pm 4 \mathrm{msec}$ in measured response times and a negative bias of at least $9.2 \mathrm{msec}$ in recorded times (keyboard scanning time plus a constant transmission time of $1.2 \mathrm{msec}$ ). Graves and Bradley (1987) tested an IBM PC keyboard and reported a mean error of $18.3 \mathrm{msec}$, with a standard deviation of $4.3 \mathrm{msec}$.

In contrast to the keyboard, the state of the parallel port can be read directly by the PC without the concomitant scanning and transmission delays. Thus a more accurate measure of subjects' response times can be obtained using switches connected to a parallel port. When four or fewer switches are required, a PC-compatible printer port can provide the necessary interface. A schematic diagram for a simple switch box for use with a printer port is presented in Figure 1. The switches are debounced using simple resistor-capacitor integrators. This form of debouncing results in a constant negative bias of $7 \mathrm{msec}$ in recorded times. A four-button version is presented; versions with two or three buttons can be constructed by simply ignoring the portion of the diagram connecting to pins 17 (for three buttons) or 16 and 17 (for two buttons) of the printer port.

Printer Por Swith System for IBM PC family computers

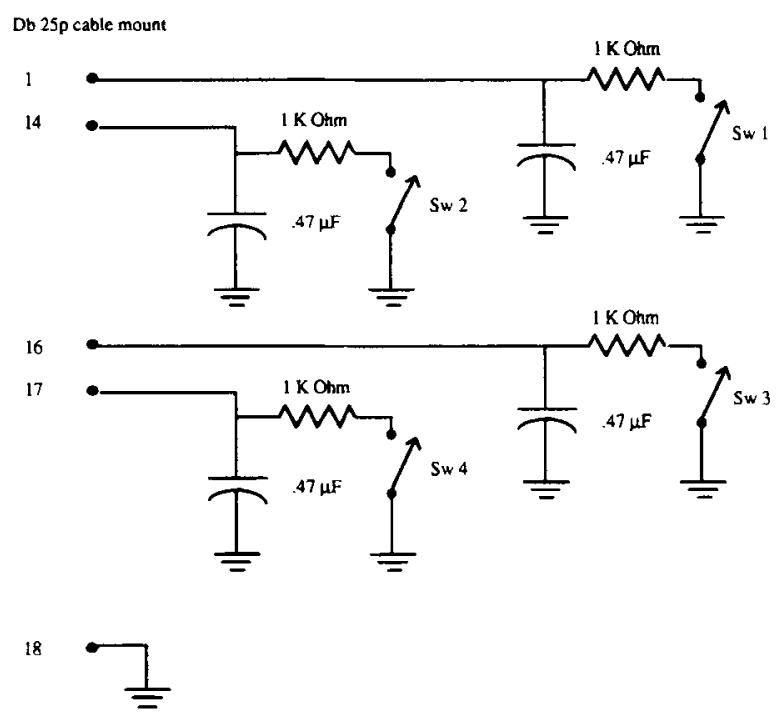

Figure 1. Schematic diagram for a general-purpose response switch that can be used to connect up to four response buttons to the printer port of an IBM PC computer. The switch features built-in debouncing of button presses, and it may be used with a variety of actual buttons or other devices.
TIMER.COM: A Flexible, Transparent

Implementation of Multiple Millisecond Timers

PC-family computers use a 16-bit counter (one third of an Intel 8253/8254 timer chip or an equivalent custom circuit) to generate hardware interrupts at a constant rate. The counter is loaded with a known value and allowed to count down at a fixed rate. When the count reaches 0 , a hardware interrupt is issued and the counter reloads itself to repeat the process. In order to maintain constant timing independent of processing speed, all PC computer timers count at $1.190 \mathrm{MHz}$. Normally, the timer interrupt is used by DOS to schedule time-dependent taskstypically, updating the time-of-day clock and controlling floppy disk drive motor shutoff. PC BIOS timer routines use a timer value of 65536 , the maximum value that can be stored in a 16-bit counter. This provides an interrupt rate of $55.2 \mathrm{msec}$.

TIMER is an assembly-language program that implements a number of useful timing functions on IBM PC computers (see Listing 1). It is a terminate-but-stayresident (TSR) program. The software interface to TIMER uses the DOS function call mechanism; thus TIMER can be used with any high-level language that can generate system calls.

TIMER increases the timer interrupt rate to one per millisecond by decreasing the value loaded into the timer chip from 65536 to 1193 . Allowing the operating system timer interrupt service routine (ISR) to run at the increased rate would affect the other time-dependent operations described above, which assume that timer interrupts will occur at the standard rate. TIMER solves this problem by emulating the standard timer interrupt. It passes control to the operating system timer ISR at nearly the expected rate ( 55 vs. $55.2 \mathrm{msec}$ ).

Running TIMER.COM from the DOS prompt installs TIMER's resident code and inserts its ISRs in the system timer and DOS function interrupt chains. The resident portion of TIMER occupies approximately $1 \mathrm{~K}$ of memory. For most high-level languages, access to TIMER is easiest and fastest using a language-specific assembly-language interface module, which translates function calls from the calling convention of the high-level language into the format required by TIMER.

TIMER implements five timers with $1-\mathrm{msec}$ resolution. Two are general-purpose 32-bit timers; two implement periodic repeating interval functions (such as A/D conversion at a regular rate); and one provides timing for parallel-port connected switches and for programmed pauses. Each can be started and read independently. TIMER and its interface modules provide the following: (1) the ability to start and stop TIMER completely; (2) access to two independent, 32-bit millisecond timers, whose maximum timable period is about 2.3 million seconds; (3) repeating interval timers, which are used to schedule regularly repeating events; (4) the ability to measure response time using switches attached to a printer 
port; and (5) programmed pauses of specified duration. These functions have proved to be adequate for a variety of experimental tasks in our laboratory, including running standard chronometric and semantic priming experiments and managing external devices such as videotape controllers.

Interface modules have been developed for five widely used programming languages. Listings $2-5$ present interface modules and corresponding function-definition files for use with Microsoft C, Microsoft FORTRAN, Microsoft QuickBASIC, and Borland Turbo Pascal. ${ }^{1}$ All but the Turbo Pascal interface, which is written in Turbo Pascal, are written in assembly language.

Comparison with previous programs. A number of approaches to obtaining millisecond resolution timing on IBM PC computers have been described previously. Some approaches require additional hardware (Segalowitz, 1987, 1988). Crosbie (1989) has described a very different approach, based on directly reading the value of the latched timer, which could theoretically produce timing to accuracies of about a microsecond. For most purposes, timing resolution of $1 \mathrm{msec}$ is a reasonable standard that avoids the added complexity of dealing with large values and inconvenient units of time.

The strategy of reprogramming the system timer to produce millisecond-level interrupts has been described previously. Several programs exist that reprogram the timing interrupts as TIMER does, but they do not pass the expected 55-msec interrupt back to the operating system (Bührer, Sparrer, \& Weitkunat, 1987; Dlhopolsky, 1988; Graves \& Bradley, 1987, 1988). This can cause difficulties for other routines that expect timing interrupts to occur at the standard rate (e.g., this interrupt is used to turn off power to the floppy disk drive's motor after read/write operations), as well as cause unnecessary processing to occur. The entire DOS timer ISR is executed every millisecond rather than every $55 \mathrm{msec}$. TIMER overcomes these problems by limiting the processing that occurs each millisecond, and by passing control to the operating system ISR at the expected interval. Previous timing programs have also tended to be language-specific, to BASIC (Bührer, Sparrer, \& Weitkunat, 1987; Graves \& Bradley, 1987, 1988), Turbo Pascal (Crosbie, 1989), or C (Dlhopolsky, 1988). TIMER's design allows it to be readily used with any language that can call operating system services, either through an assembly-language routine or by using facilities native to the language itself. TIMER's TSR design allows it to be isolated from the user's program. Because it is nearly impossible for the higher level program to disrupt TIMER, timing functions and system integrity are not vulnerable to bugs in user programs.

In the remainder of the paper, we will describe two uses of TIMER. The first involves a strategy for measuring and minimizing errors in the timing of events that takes advantage of the availability of multiple timers. The second consists of a benchmark program for measuring the time a given computer takes for performing a variety of tasks relevant to controlling psychological experiments, and reports results for a number of different computers using the basic IBM PC design.

\section{A Strategy for Measuring and Minimizing Timing Errors}

Experimental tasks, particularly those involving small intervals between the presentation of successive stimuli (SOAs), consist of both stimulus processing tasks that must be accomplished and intervals that must be timed. Often both must be accomplished at the same time, and the experimenter must adjust time intervals to take into account time required to generate stimuli. Listing 6 presents an example of how one might accomplish this, using TIMER from a higher level language. The example program, MICROMAN.C, is written in C, although the same strategy could be used with other higher level languages. The basic procedure involves (1) starting both timers; (2) performing some work in preparing a stimulus display; (3) reading the first timer; (4) waiting for an interval that represents the best estimate of the remaining time to stimulus display [(desired interval) - (elapsed time) - (screen refresh interval)/2]; (5) presenting the stimulus on the next screen refresh; and (6) recording the actual elapsed time by reading the second timer.

The last step-recording the actual elapsed time-may seem redundant, and in most cases it will prove to be so. It is a simple and useful precaution, however, which can be used to provide better control over the interval used in Step 4 above if this is desired. The screen refresh rate imposes limits on the values that can be used for SOAs. In the worst case, in which the total elapsed time is within the range of variation of screen retraces, it is possible that the actual SOA will vary by one screen refresh interval (16.7 msec on PC ATs with EGA graphics). The interval used in Step 4 can be adjusted to eliminate any problem of synchronization between SOAs and screen refresh times. Because of the limitations imposed by screen refresh rates, actual SOAs will vary by as much as one refresh interval from the nominal rate imposed by the experimenter. Recording this rate will reveal if there is any problem of variation in SOAs across trials, as discussed above, and will indicate what the constant error is between desired and actual SOA.

MICROMAN.C introduces a random variation of $100 \mathrm{msec}$ in the interval between presentation of successive screens, then uses TIMER to adjust for this variation. In 100 trials on an 8-MHz IBM AT, with a desired SOA of $300 \mathrm{msec}$, the actual SOA varied from 299 to $300 \mathrm{msec}$. The same test on a PS/2 Model 80 produced a consistent SOA of $304 \mathrm{msec}$.

\section{PSYBENCH: A Benchmark Program for Experimentally Relevant Processes}

The second application of TIMER described here is a C program, PSYBENCH.C, which measures the time required to perform a number of tasks relevant to present computer-controlled experiments. These are: (1) loop computation (a basic measure of integer processing speed, 


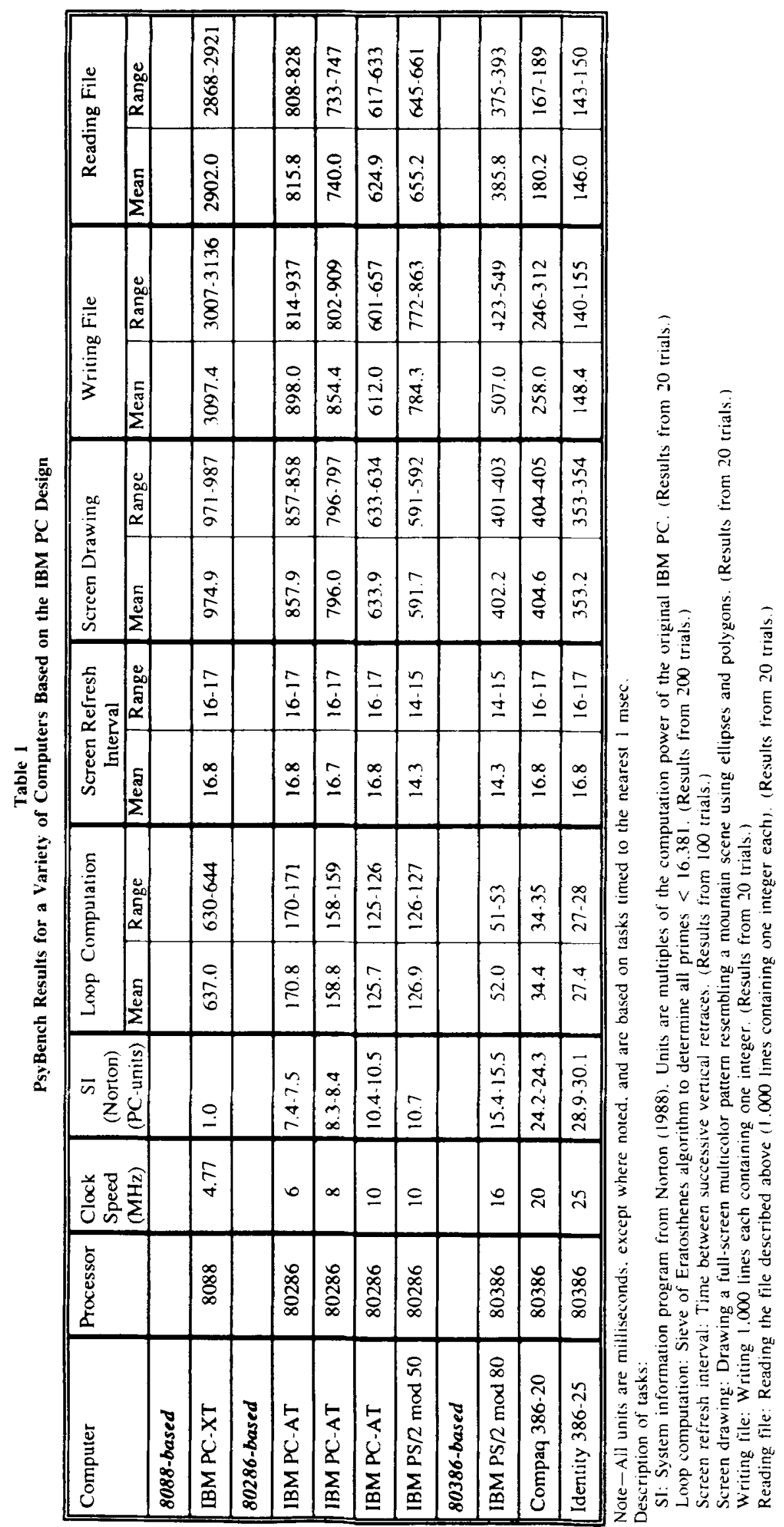


using the sieve of Eratosthenes procedure for finding prime numbers, with code from Gilbreath and Gilbreath (1983); (2) screen retrace interval (interval between successive screen retraces); (3) stimulus generation (time to generate a variety of graphics screens varying in complexity); and (4) file I/O (time to read and write standard files). PSYBENCH is presented in Listing 7. It requires an IBM PC computer (including most clones) supporting EGA graphics (including VGA graphics devices and a number of other systems that emulate EGA graphics). PSYBENCH has two main uses. First, it can ensure that a particular computer can perform these tasks with reasonable speed and reliability, which may be important in selecting a computer for experimental use. Second, it provides a meaningful benchmark of the time a particular computer requires relative to others in performing the kinds of tasks required for running psychological experiments. PSYBENCH results for a number of computer systems available at the Psychology Department of the University of Texas are presented in Table 1. All computers were running MS-DOS Version 3.3. For purposes of comparison, results from the system information program (SI.COM) from Version 4.5 of the Advanced Edition of the Norton utilities (Norton, 1988) are presented. This is comparable to the loop routine in PSYBENCH in being a measure of basic processing speed, but note that SI provides a speed index rather than a time measurement. PSYBENCH prints out additional information about specific subtasks; these results are provided with the program.

Results from running PSYBENCH on a number of different computers show that in general the tasks assessed vary substantially between different systems, but that they tend to be reliable to within measurable limits $( \pm .5 \mathrm{msec})$ on any particular computer. The obvious exception to this result concerns disk $\mathrm{V} / \mathrm{O}$. This is not very surprising, given the flexibility with which data sectors can be assigned to physical disk locations in PC-compatible computers. It is worth noting as a general point that although these benchmark tasks are similar to tasks used in many psychological experiments, these results might not be predictive for any given experimental program and computer. This is particularly true for the file I/O results, which can be greatly affected by the overall state and capacity of the disk at the time that $\mathrm{I} / \mathrm{O}$ occurs.

IBM PC computers have become a ubiquitous tool for experimental psychologists. The design of these devices causes problems for experimenters desiring to control the presentation of experimental events accurately, but they are also designed such that it is possible to measure and minimize discrepancies between desired and actual times of these events. The capacity of computers sharing this general design has also evolved substantially (as the results in Table 1 demonstrate). Strategies for ensuring comparability of the timing of experimentally important events across computers differing in speed and capability will become, if anything, more critical over time.

\section{Program Availability}

A disk containing source and executable versions of the programs described here, additional documentation for TIMER and more detailed benchmark results can be obtained by sending a check for $\$ 15$ to Kevin F. Miller. A version of TIMER that works with Microsoft Windows will also be included upon request. Please specify disk format ( 3.5 or 5.25 in.). Individuals who have access to electronic mail can receive copies from Kevin F. Miller at kmiller@emx.utexas.edu or PSHU654@UTA3081.

\section{REFERENCES}

Barnhart, C. L. (1984). The Barnhart dictionary companion (Vol. 3, No. 4). Cold Spring, NY: Author.

Bührer, M., SPARRER, B., \& WeitKunat, R. (1987). Interval timing routines for the IBM PC/XT/AT microcomputer family. Behavior Research Methods, Instruments, \& Computers, 19, 327-334.

Crosbie, J. (1989). A simple Turbo Pascal 4.0 program for millisecond timing on the IBM PC/XT/AT. Behavior Research Methods, Instruments, \& Computers, 21, 408-413.

DLhopolsKY, J. G. (1988). C language functions for millisecond timing on the IBM PC. Behavior Research Methods, Instruments, \& Computers, 20, 560-565.

DLhopolsky, J. G. (1989). Synchronizing stimulus displays with millisecond timer software for the IBM PC. Behavior Research Methods, Instruments, \& Computers, 21, 441-446.

DoNDERS, F. C. (1969). On the speed of mental processes (W. G. Koster, trans.). Acta Psychologica, 30, 412-431. (Original work published 1868)

Gilbreath, J., \& Gilareath, G. (1983). Eratosthenes revisited. Byte, 8, 283-326.

Graves, R., \& Bradley, R. (1987). Millisecond interval timer and auditory reaction time programs for the IBM PC. Behavior Research Methods, Instruments, \& Computers, 19, 30-35.

Graves, R., \& Bradley, R. (1988). More on millisecond timing and tachistoscope applications for the IBM PC. Behavior Research Methods, Instruments, \& Computers, 20, 408-412.

LeMAy, H., LeRner, S., \& TAYLoR, M. (1988). The facts on file dictionary of new words. New York: Ballantine Books.

Norton, P. (1988). The Norton utilities (Version 4.5). Santa Monica, CA: Peter Norton.

Paredes, D. R., Miller, K. F., * Creeger, C. P. (1990). Graphic precision: Displaying graphical stimuli in real time on IBM PC family computers. Unpublished manuscript, University of Texas at Austin, Department of Psychology.

Posner, M. I. (1978). Chronometric explorations of mind. Hillsdale, NJ: Erlbaum.

Segalowitz, S. J. (1987). IBM PC Tachistoscope: Text stimuli. $B e-$ havior Research Methods, Instruments, \& Computers, 19, 383-388.

SEgalowitz, S. J. (1988). IBM PC tachistoscope: II. Assembly language subroutines. Behavior Research Methods, Instruments, \& Computers, $20,432$.

\section{NOTE}

1. Interface modules for IBM Professional FORTRAN and IBM/Microsoft Pascal are not presented, but are included with the other modules on the disk available from the authors. 
Comment $/^{*}$

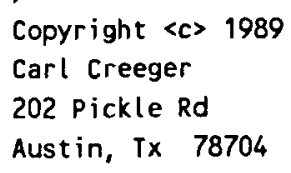

This Program may be distributed with out charge for any no-profit use but may not be sold.

No Warranty, expressed or implied applies to this program.

Timer is accessed using Dos function calls. Parameters and return values are passed to $T$ imer by Language interface routines. These routines are supplied as object modules and are linked with the user code. Executing Timer.Com installs Timer code, inserts the timer service routine and Timer's timer action interrupt service routine into their respective interrupt chains. The resident portion of timer occupies about 1.2 kbytes of memory. When active (no timers enabled), the timer action interrupt service rout ine consumes about $5 \%$ of cpu time on a stock AT. The language interface routines and the timer service interrupt service routine use the following parameter passing convention:

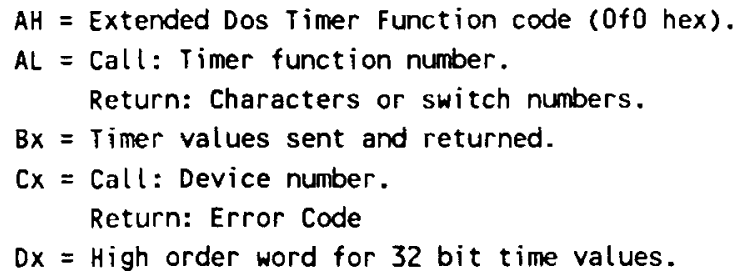

Timer provides the following function groups and functions:

TIMER CONTROL FUNCYIONS:

SIART, function number 1

Activates the Timer's Interrupt Service Rout ine and sets the system clock interrupt period to $1 \mathrm{~ms}$. Setup must be called before any other timing rout ines are used. Parameters: none.

Return: none.

STOP, function number 2

Undoes what start does. Restores the system timing routines to normal. This routine should be called before the program exits or the system time be somewhat inaccurate. Parameters: none.

Return: none.

ACKNOWLEDGE, function number 3

Timer uses this function to determine whether it has already been installed.

Returns offh in AL, 0 in $A H$.

REPEATING INTERVAL TIMER (TYPe 2 Timer) FUNCTIONS:

DINIT, function number 4

Start a repeating timed period and return. Two timers are available, 1 and 2 . Parameters: Device selected in $\mathrm{CX}$, Time in $\mathrm{BX}$

Return : illegal device returns 2 in $c x$.

WAIT, function number 5

Holds execution until the end of the next repeating interval of a Type 2 timer.

Parameters: $\mathrm{CX}=$ device number. 
Listing 1 (Continued)

Return: Error 2, bad device number, error 3, selected delay timer has not been initialized by dinit, error 8 wait over-run.

REPORT FLAG, function number 6

Get a Type 2 timer's event flag, I.E. has the next interval elapsed or not.

Parameters: $C X=$ device number .

Return : 16 bit logical flag in dx, same errors as function WAIT.

CONTINUOUS TIMER (TYPe 1 Timer) FUNCTIONS:

START_T, function number 7

Initializes a 32 bit timer, clears the timer and resets the overflow flag.

Parameters: Device number in $\mathrm{CX}$.

Returns: Error on illegal timer number.

TIMER, function number 8

Returns the present value of the specified 32 bit timer.

Parameters: Device number in $\mathrm{CX}$.

Returns a 32 bit integer time value in DX:BX. Errors, bad timer number and timer overflow.

ASSORTED TIMING FUNCTIONS

STIME, function number 9

Measure response time to a switch press. Switches are attached to a printer port.

Parameters: Time-out value in $\mathrm{BX}$.

Returns : response time in $\mathrm{BX}$, switch number in al. $\mathrm{CX}=4$ means no switch press was

detected ( $T$ imed out).

PAUSE, function number 10

Function: Delay for the time specified in parameter.

Parameters: Delay, in BX, time to wait in milliseconds.

Return: none.

SET_SWITCH_PORT, function number 11

Select the printer port used by stime (LPT1 - LPT3). The default port is LPI1.

Parameters : $C X$ is set to LPT\# (1-3 allowed).

Returns : Error 2, illegal port \#, error 9, requested port not installed.

TIMER ERROR RETURNS:

The following error are reported:

$c x=1, T$ imer not installed. This error code is returned by the interface routine. Timer returns $A H=0$. The interface routine detects $A H=0$ returns the error code.

$c x=2$, invalid device error

$c x=3$, wait, call to un initialized timer.

$c x=4$, stime timed out

$c x=5$, timer overflow

$c x=6$, bad function number

$c x=7$, Timer not started

$c x=8$, wait over-run.

$c x=9$, bad device number in call to set_switch_port.

end of comment */ 


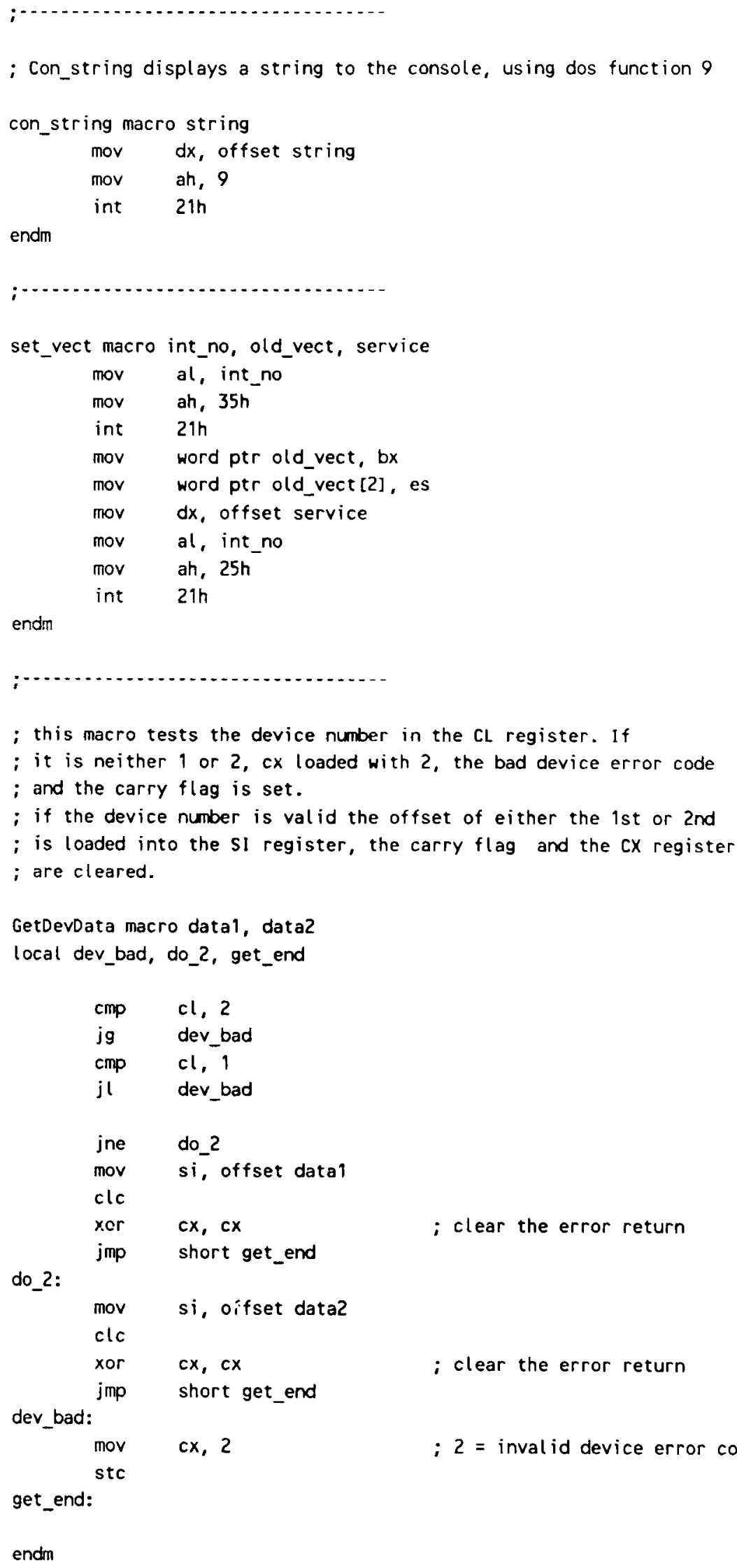




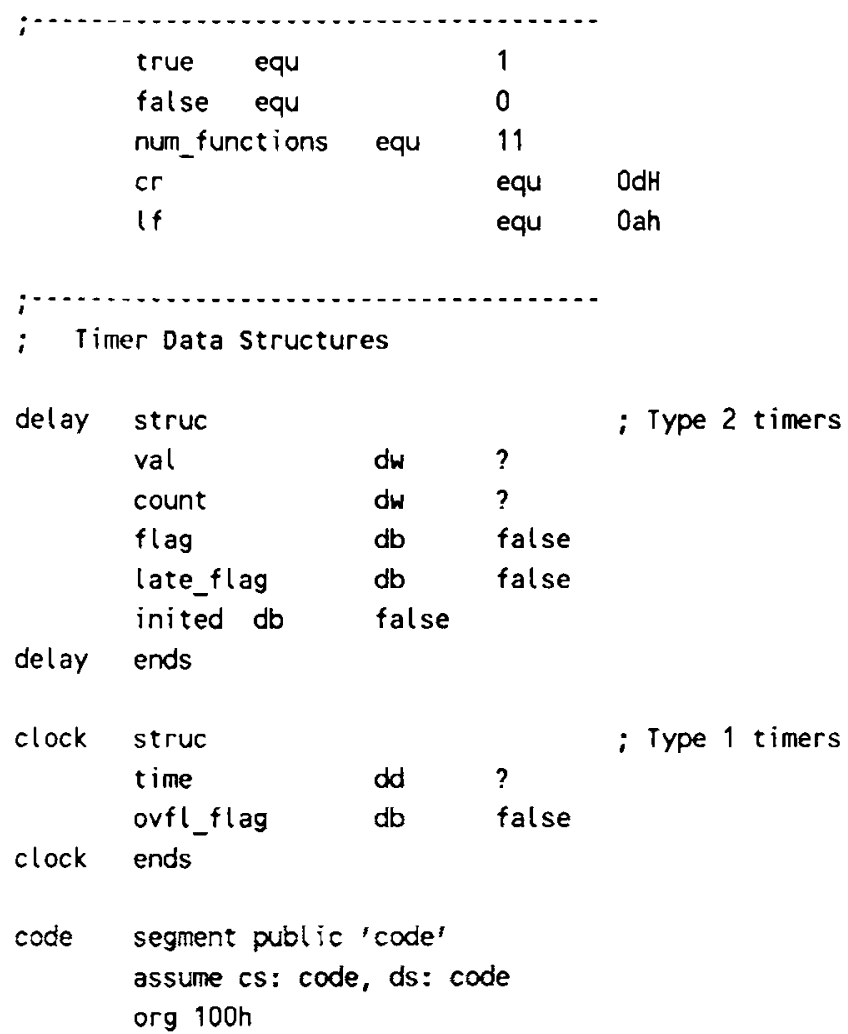




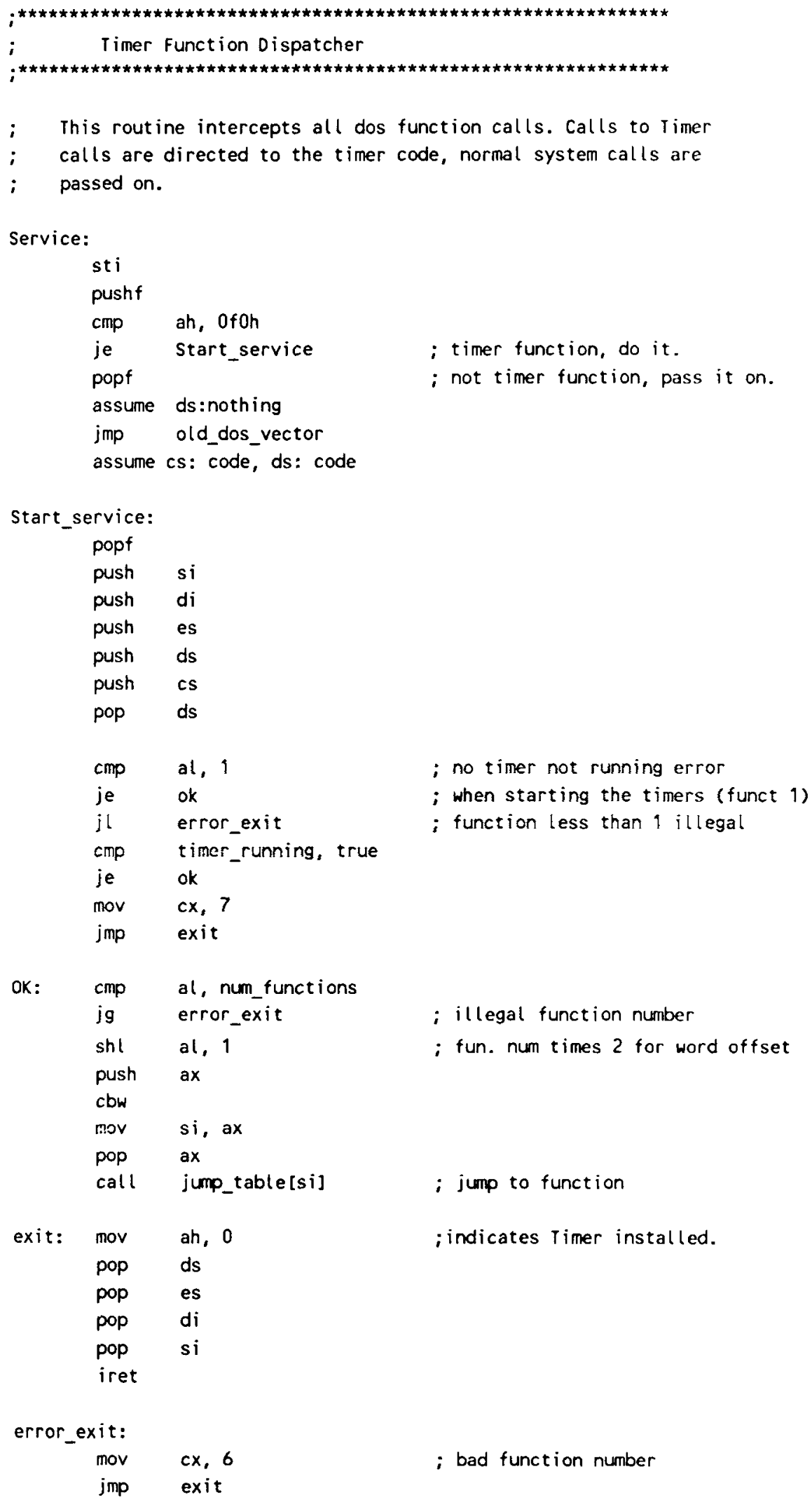




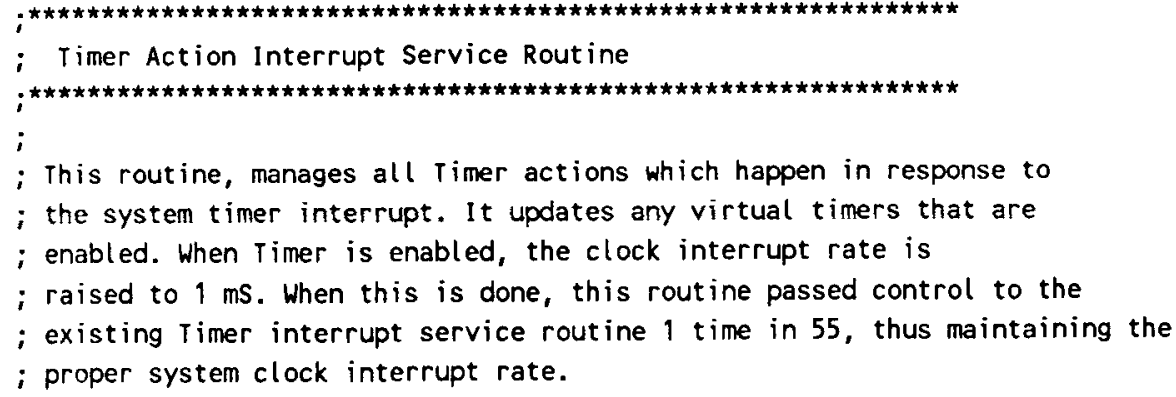

ISR:

$\begin{array}{lll}\text { sti } & & \text {; re-enable interrupts } \\ \text { push } & \text { ax } & \text {; save and setup } \\ \text { push } & d s & \\ \text { mov } & a x, c s & \\ \text { mov } & d s, \text { ax } \\ \text { cmp } & \text { timer_running, true } & \\ \text { je } & \text { running } & \\ \text { jmp } & \text { inactive_exit }\end{array}$

running:

inc up_count

; up counter for stime and pause

inc word ptr timer_1.time

; type 1 timers

jnz do_timer_2

inc word ptr timer_1.time +2

jnz do_timer_2

mov timer_l.ovfl_fiag, true

do_timer_2:

inc

word ptr timer_2.time

: timer 2

inz end_timer

inc word ptr timer_2.time +2

jnz end_timer

mov timer_2.ovfl_flag, true

end_timer:

cmp

delay_1.inited, true

; type 2 timers

jne $d z$

cmp delay_1.flag, true

jne not_late

mov delay_1.late_flag, true

not late:

dec delay_1.count

jnz d_2

mov ax, delay_1.val

mov delay_1.count, ax

mov delay_1.flag, true

d_2:

cmp delay_2.inited, true

jne end_delay

cmp delay_2. flag, true

jne not_late2

mov delay_2.late_flag, true

not_late2:

$\begin{array}{ll}\text { dec } & \text { delay_z.count } \\ \text { jnz } & \text { end_delay } \\ \text { mov } & \text { ax, delay_2.val }\end{array}$


Listing 1 (Continued)

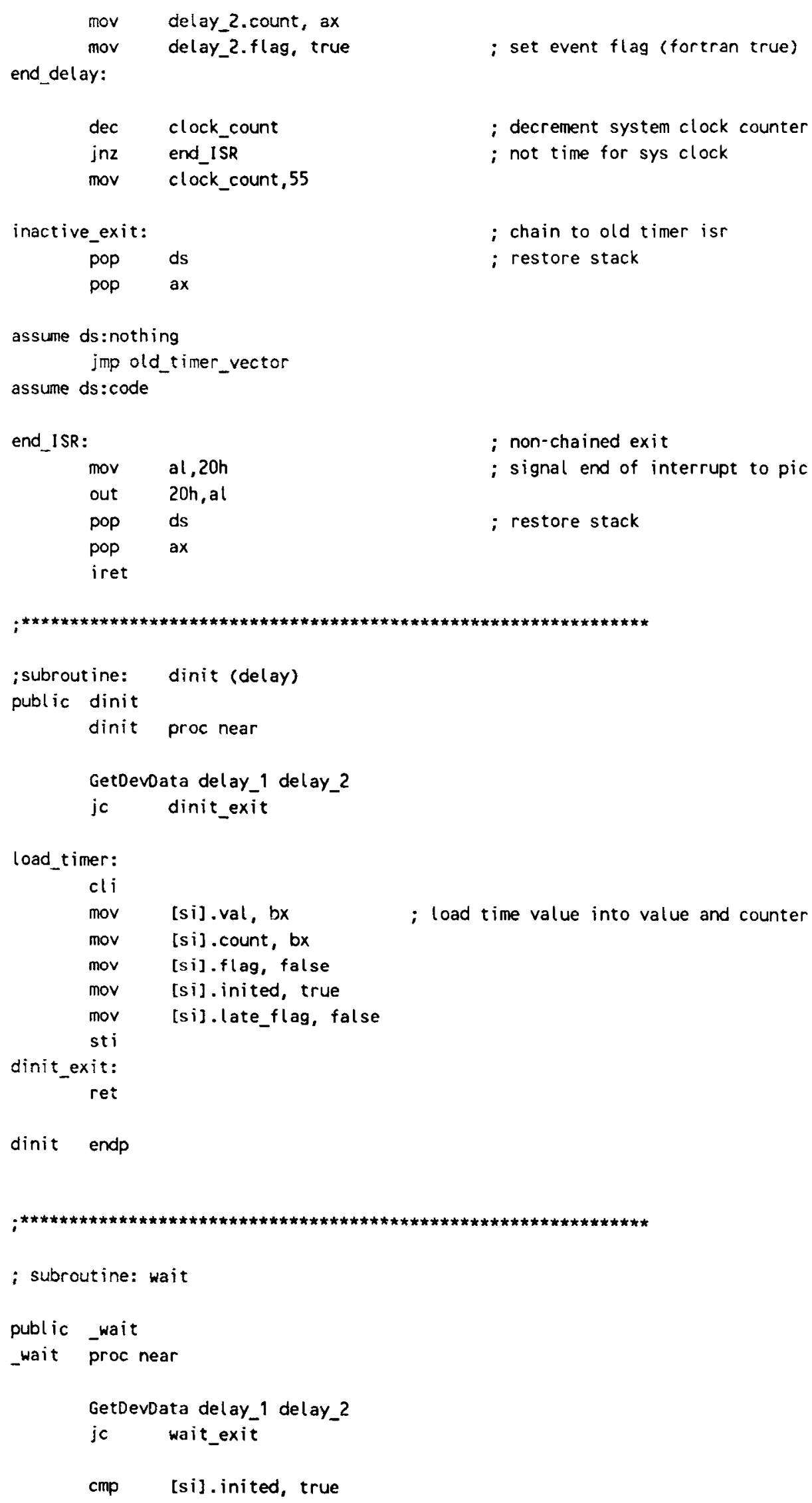


Listing 1 (Continued)

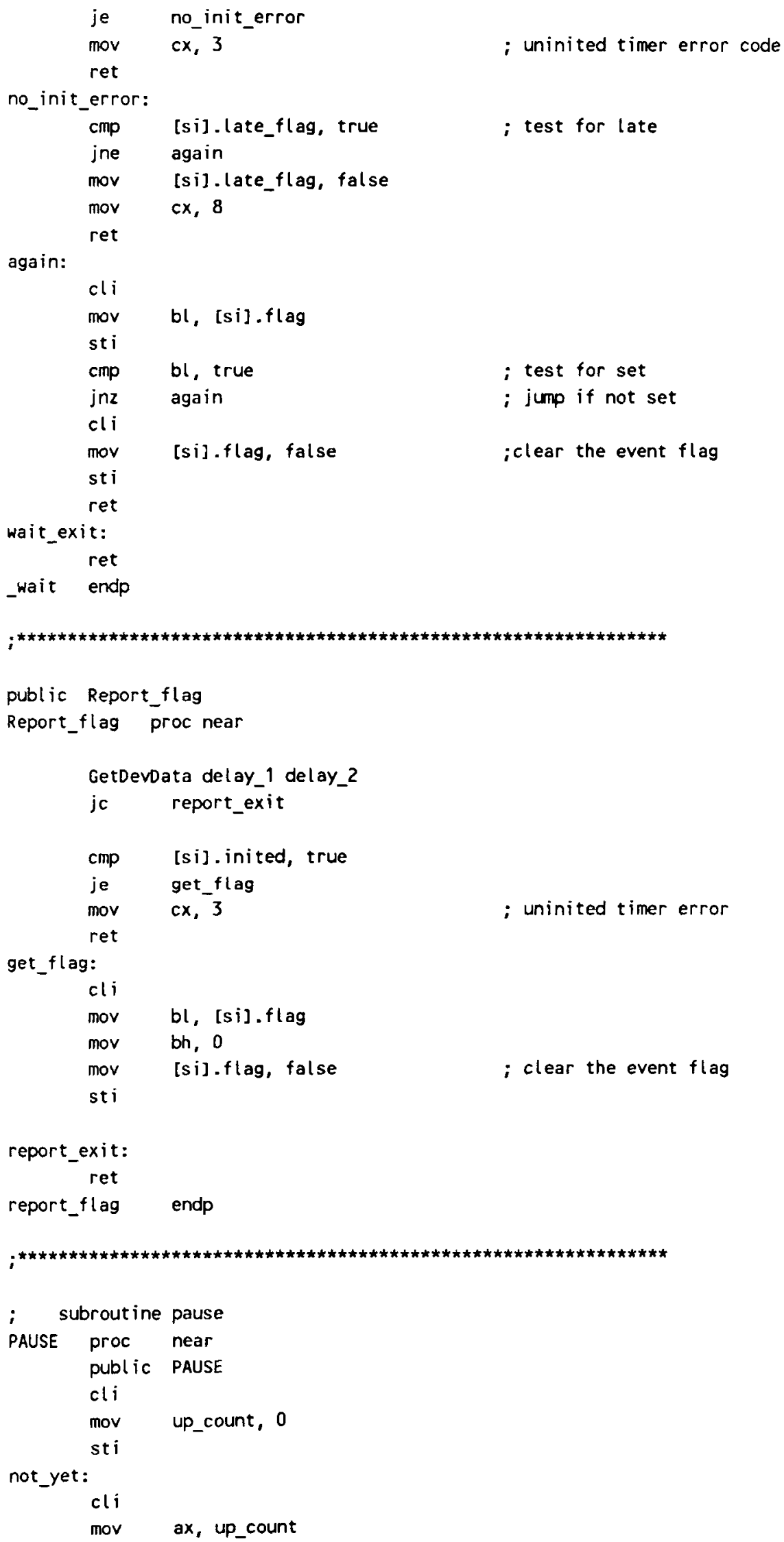


Listing 1 (Continued)

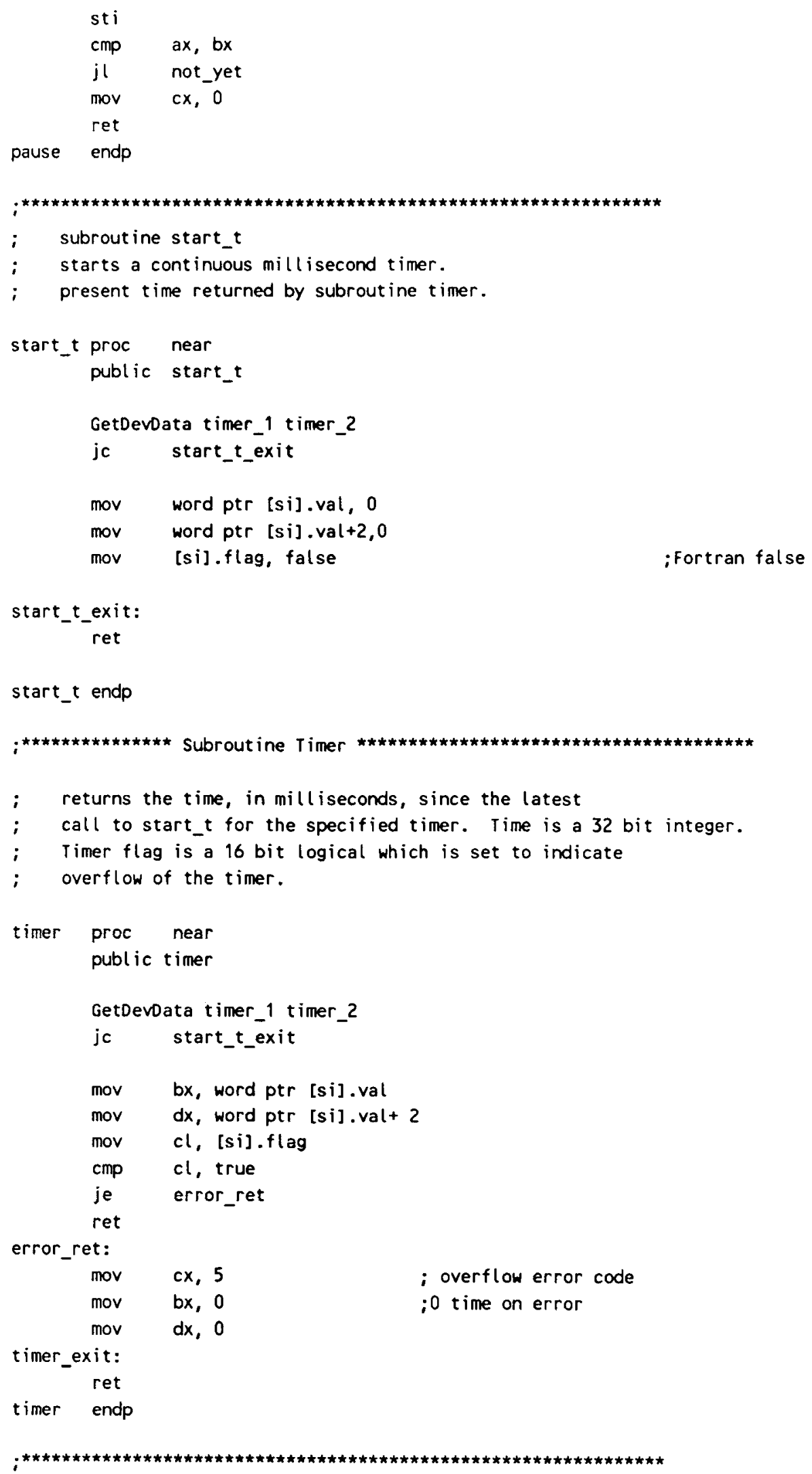


; subrout ine STIME

; This routine measures the elapsed from the time the routine call

; to the time of detection of a switch press.

; The routine returns the time (long integer) and switch number.

public stime

stime proc near

$\begin{array}{lll}\text { mov } & \text { up_count, } 0 & ; \text { zero the counter } \\ \text { mov } & d x, \text { port } & ; \text { printer control port } \\ \text { mov } & \text { al, } 20 & ; \text { int disable, bit } 3 \text { hi...... } \\ \text { out } & d x, \text { al } & ; \text { output lines high for pull up } \\ \text { itch: } & \end{array}$

read_switch:

mov $d x$, port printer control port

in $a l, d x$

and al, 15

xor al, 4

mov $c x, 1$

mov $d l, 1$

; mask off all but low 4 bits

; invert 3rd bit because of the port

; counter for switch check loop

; mask value for switch check

next_bit:

cmp al, dl

iz got_switch

shl $d l, 1$; set mask to next bit position

inc $c x \quad$; loop counter (also switch number)

cmp $c x, 4$

jle next_bit

cmp bx, up_count ; check for time out

jg read_switch ; if no time out, keep checking switches

nov $c x, \overline{4}$; else return timed out error.

got_switch:

mov al, cl switch number in al

mov ah, 0

mov $c x, 0$; clear error return

$\mathrm{cli} \quad$; read the counter

mov bx, up_count

mov $d x, 0$

sti $\quad$ i let it count again

stime endp

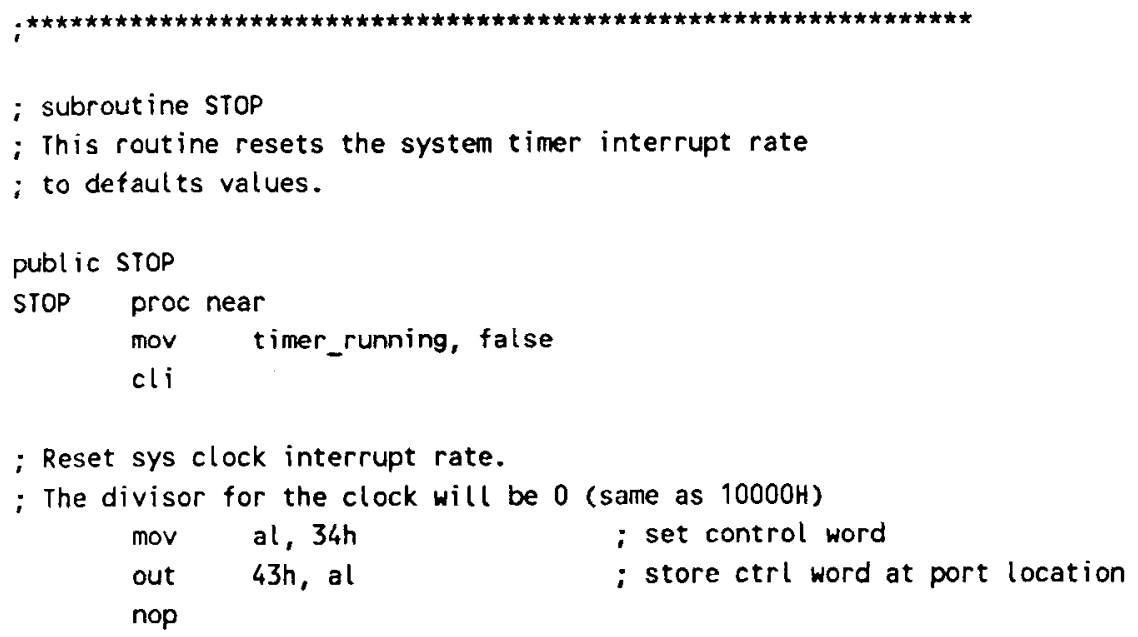


Listing 1 (Continued)

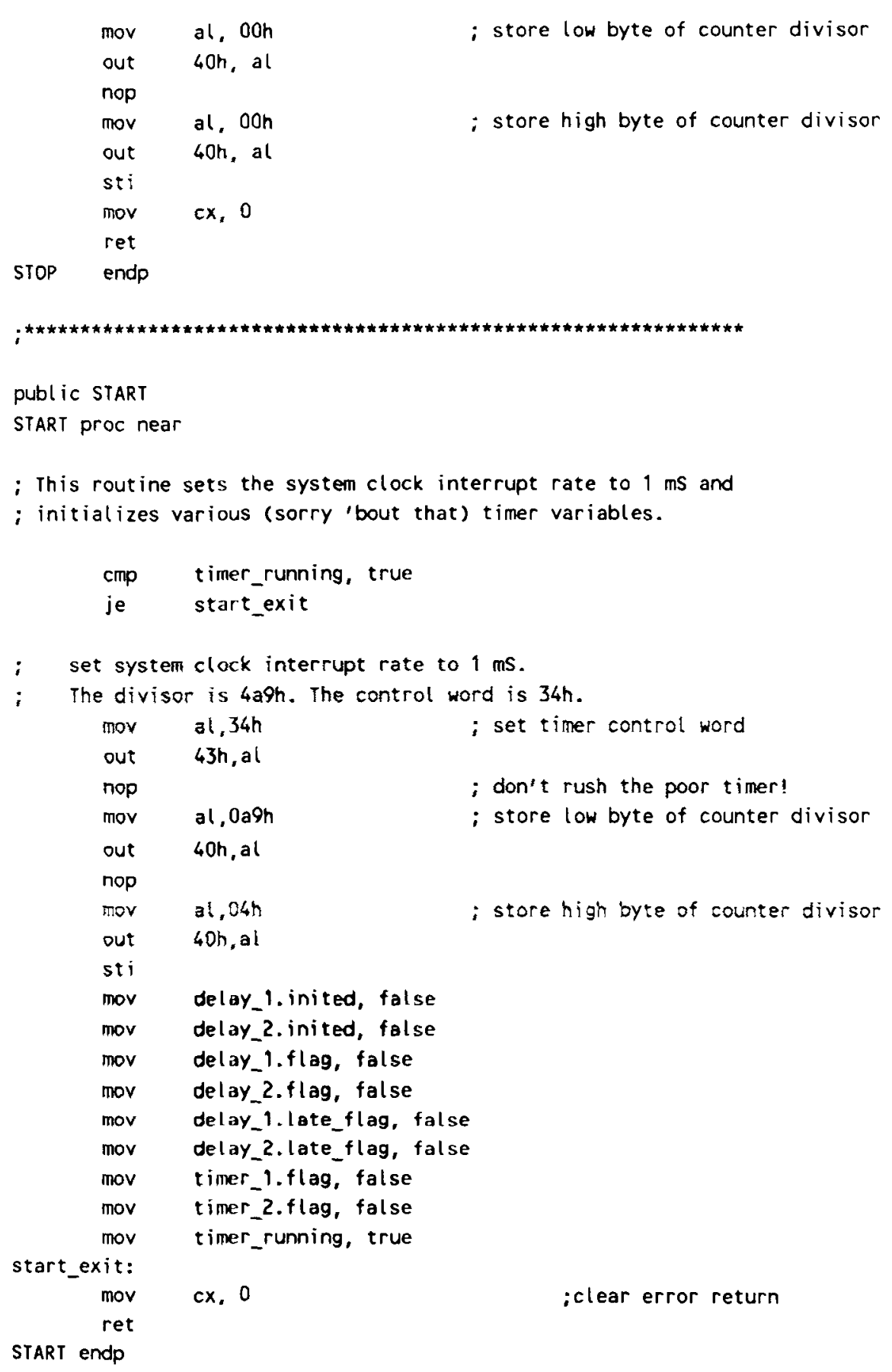

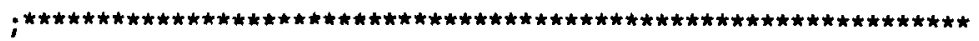

publ ic ACKNOWLEOGE

ACKNOWLEOGE proc near

mov al, offh
mov ah, 0
mov cx, 0
ret
EDGE endp

ACKNOWLEDGE endp 
Listing 1 (Continued)

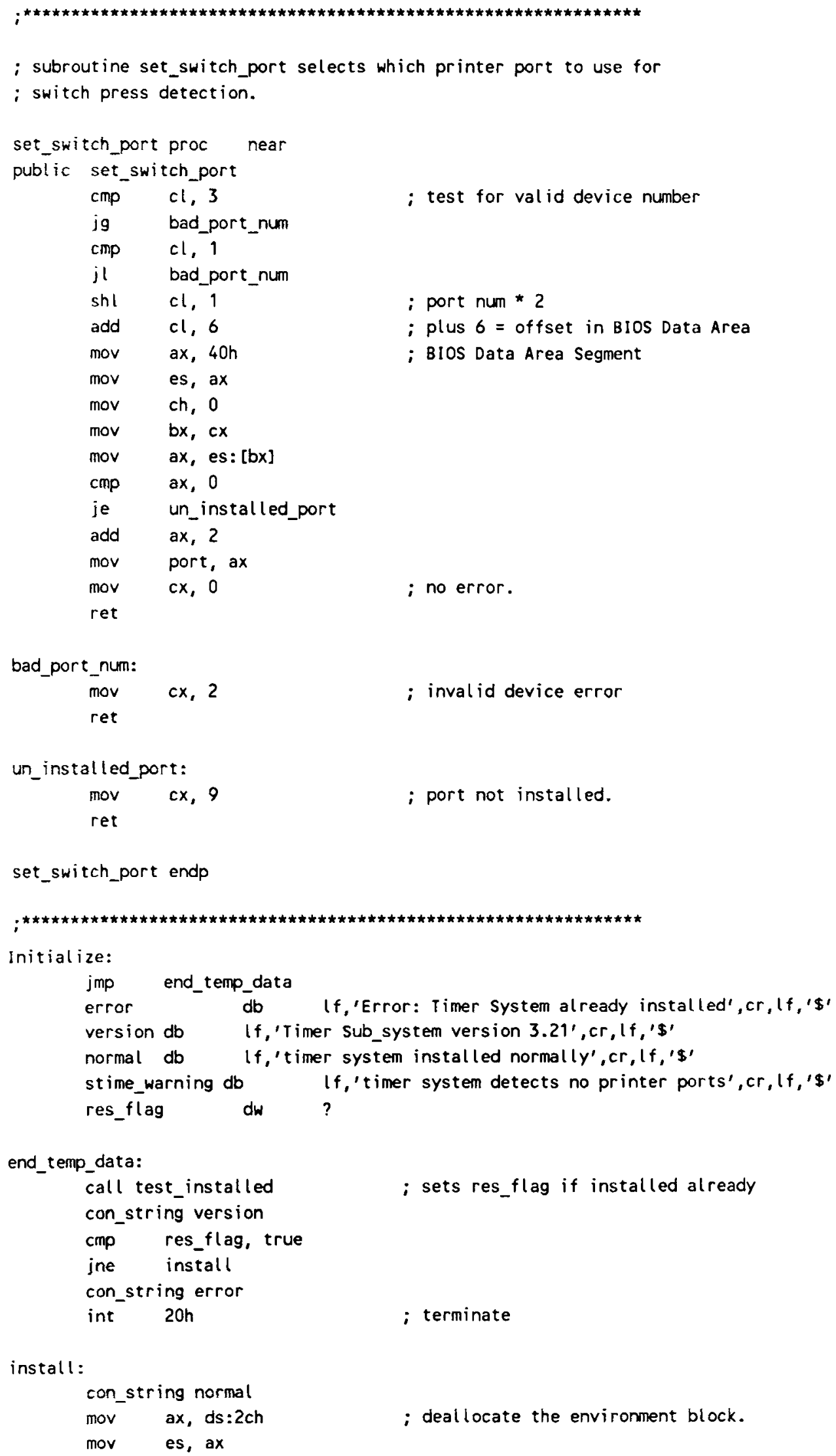


Listing 1 (Continued)

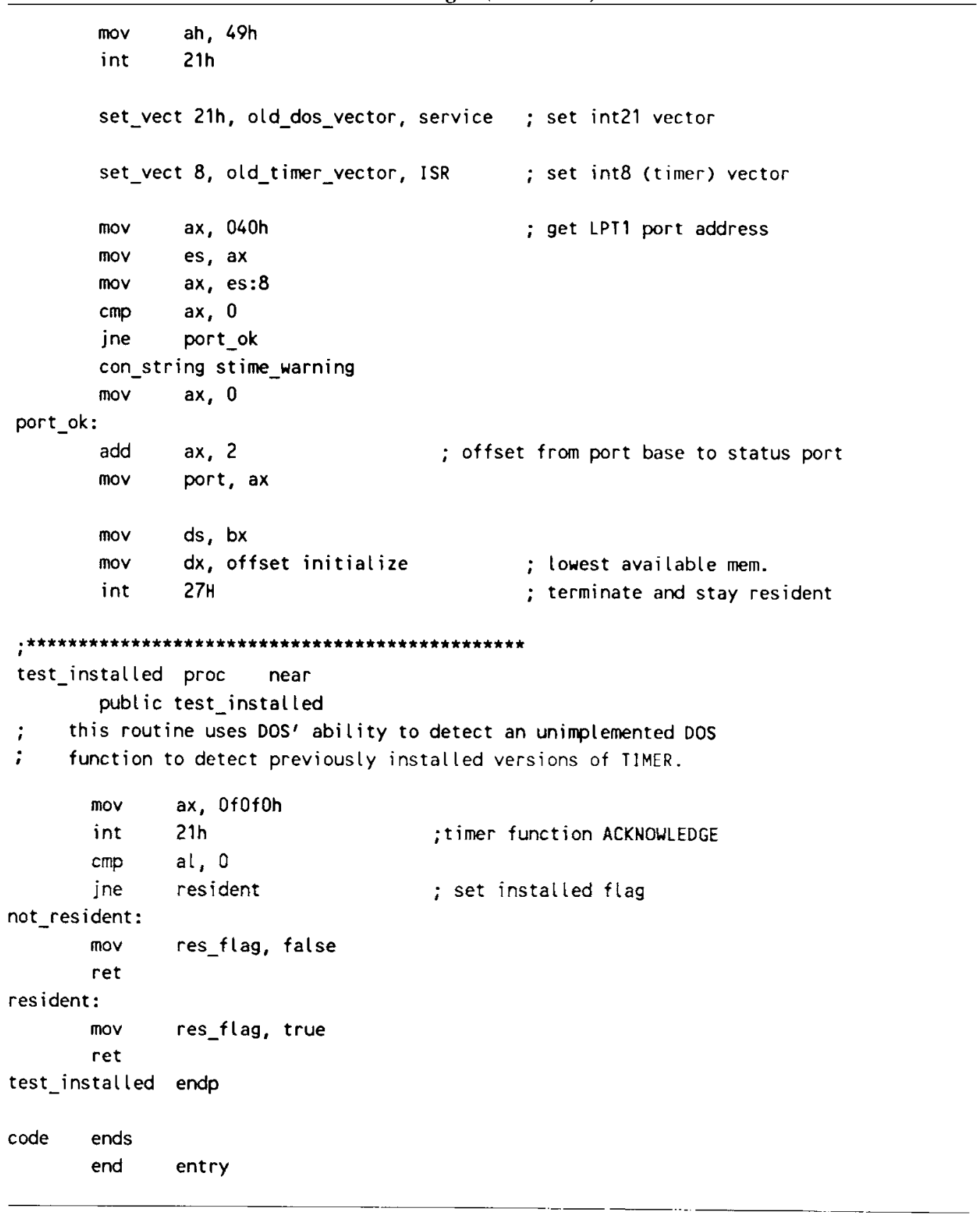

Listing 2: Microsoft $C$ Language Interface for TIMER

1. timing.h - header file to be included in C programs that use TIMER. short start(void);

short stop(void);

short dinit(short, short);

short wait(short);

short report_flag(short, short *);

short start_timer(short);

short stime ( short ${ }^{*}$, short ${ }^{*}$ );

short read_timer(short, long *);

short stall(short); 
Listing 2 (Continued)

\section{2. ctimer.asm - Microsoft C interface for TIMER.}

Copyright $\langle\mathrm{C}\rangle 1989$

Carl Creeger

202 Pickle Rd

Austin, Tx 78704

This Program may be distributed with out charge for any no-profit use but may not be sold.

No Warranty, expressed or implied applies to this program.

; Use with the header file "TIMING.H"

; Functions implemented:

start, function number 1

stop, function number 2

acknowledge, function number 3

dinit, function number 4

wait, function number 5

report FLAG, function number 6

start_timer, function number 7

read_timer, function number 8

stime, function number 9

stall, function number 10

set_switch_port, function number 11

CTIMER uses the file 'CMACROS. INC' version 1.06 or 5.0. At compile time this file

; must be in the current directory or in a directory pointed to by

the "include" environment variable. 'CMACROS. INC' is included with the

Microsoft $C$ compiler.

; 'Cmacros' allow this module to produce code for any memory model,

; depending on the command line used:

small model: masm / 0 ?memS=1/Mx ctimer,.,;

medium model: masm $/ D$ ?menth=1 $/ M x$ ctimer,.,;

compact model: masm $/ D$ ?memC $=1 /$ Mx ctimer,.,;

large model: masm $/ D$ ?memL $=1 / M x$ ctimer, , ;

; All functions return 0 if successful, otherwise a TIMER error.

; See TIMER.ASM for a list of TIMER errors.

; the following warning is normal and can be ignored:

; ctimer.ASM(99): warning A4016: Reserved word used as symbol: WAIT

; definitions to control Cmacros:

?PLM=0 ; NOT PLM (pascal and fortran) calling conventions.

?WIN=0 ; NOT Windows callable.

.$x$ list ; don't list all the macros.

include cmacros.inc

.list

Dotimer macro fnum ; code common to all timer interface functions.

LOCAL proc_exit

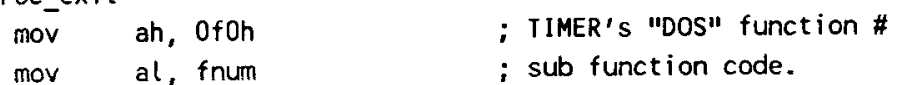

int $21 \mathrm{~h}$ 
Listing 2 (Continued)

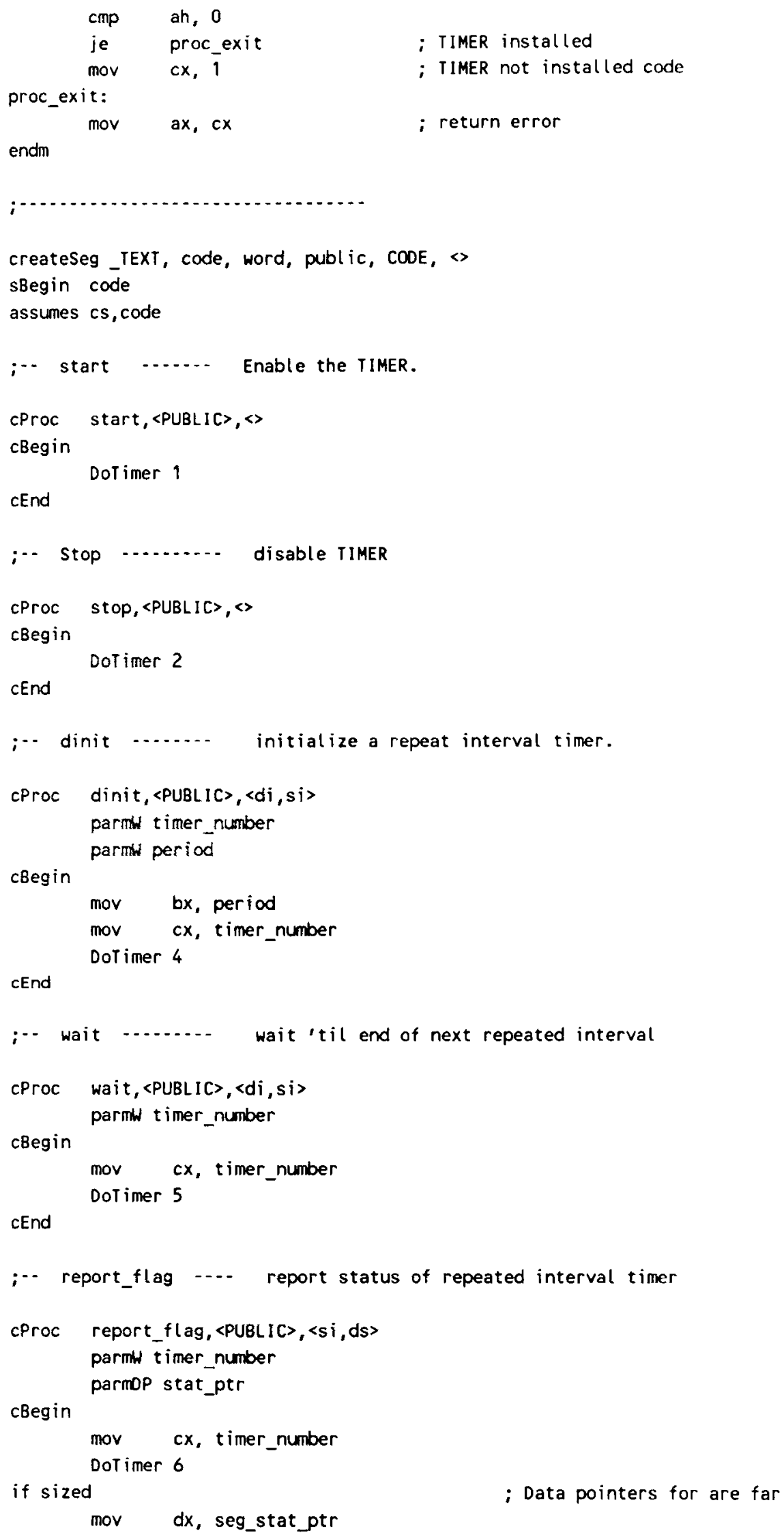


Listing 2 (Continued)

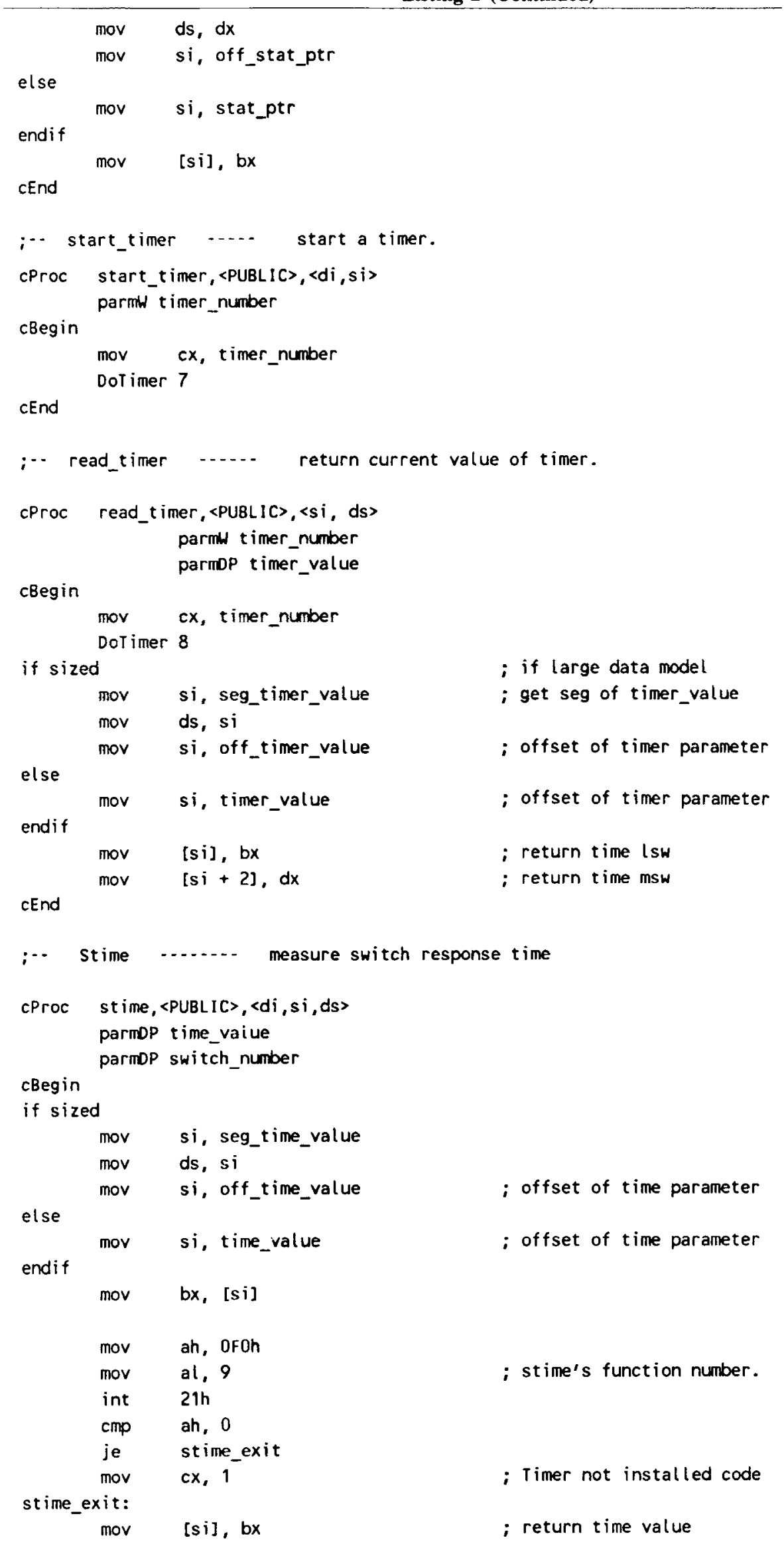


Listing 2 (Continued)

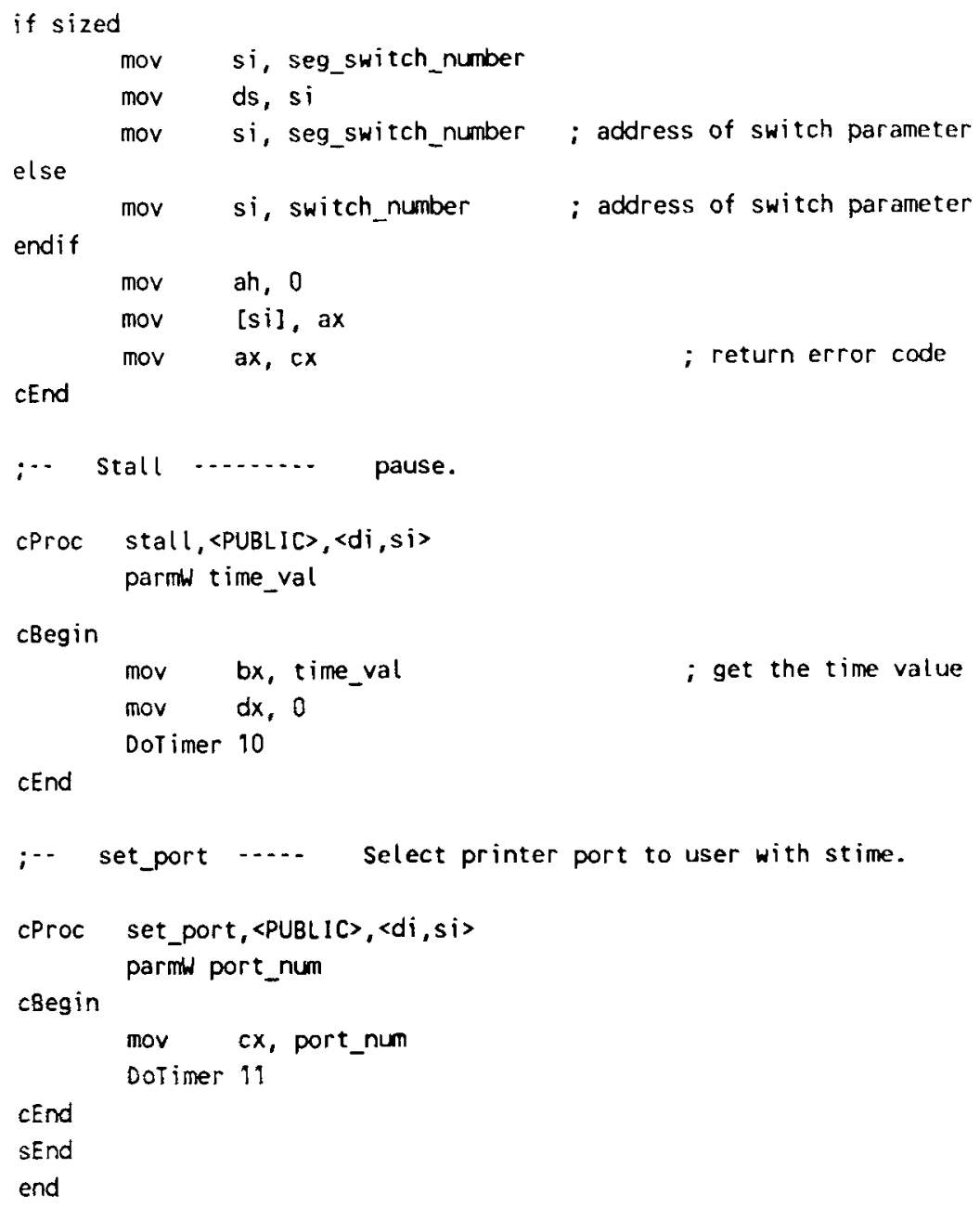

Listing 3: Microsoft FORTRAN Language Interface for TIMER

1. timer.fi - FORTRAN include file for programs that use TIMER.

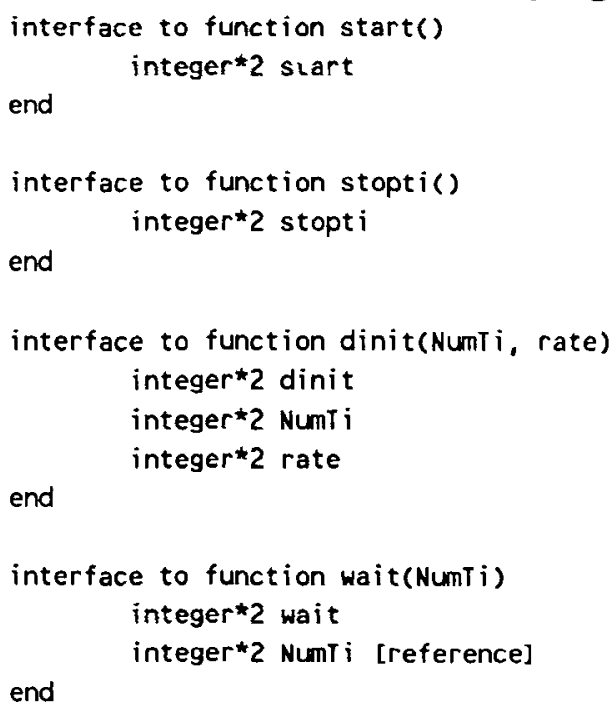




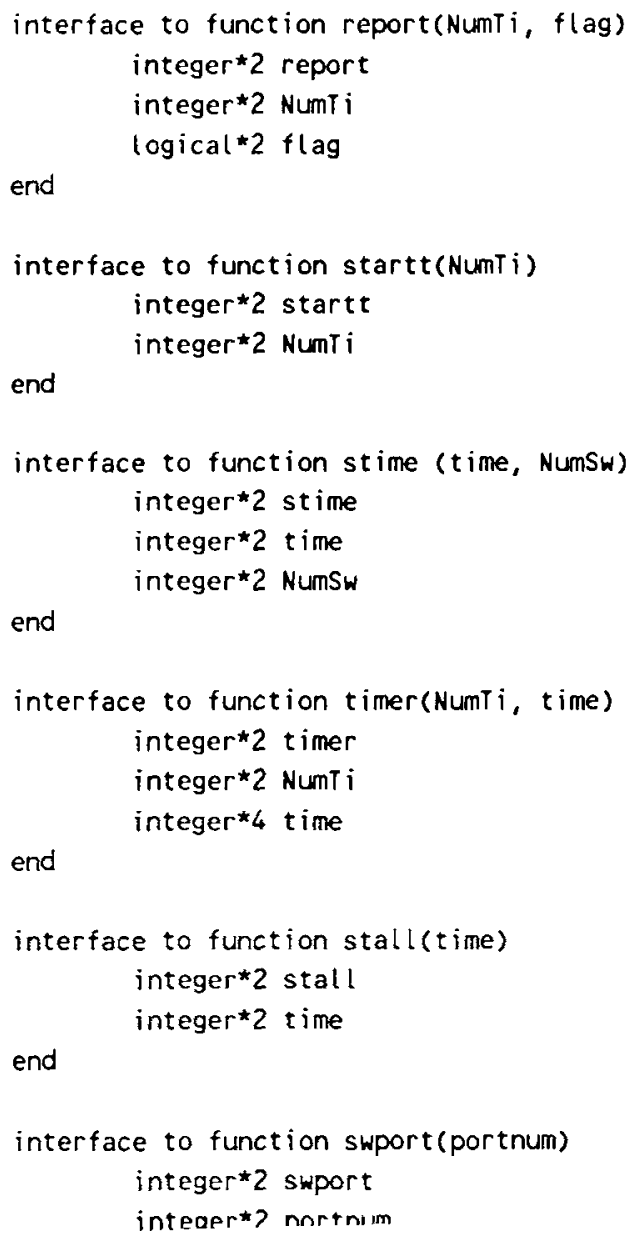

2. timer.fd - FORTRAN function definition file to be included in subroutines.
integer*2 start [EXTERN]
integer 2 stopti[EXTERN]
integer ${ }^{2}$ dinit [EXTERN]
integer 2 wait [EXTERN]
integer ${ }^{2}$ report [EXTERN]
integer*2 startt [EXTERN]
integer*2 stime [EXTERN]
inteqer ${ }^{\star} 2$ timer [EXTERN]

\section{3. msftimer.asm - TIMER interface for Microsoft FORTRAN.}

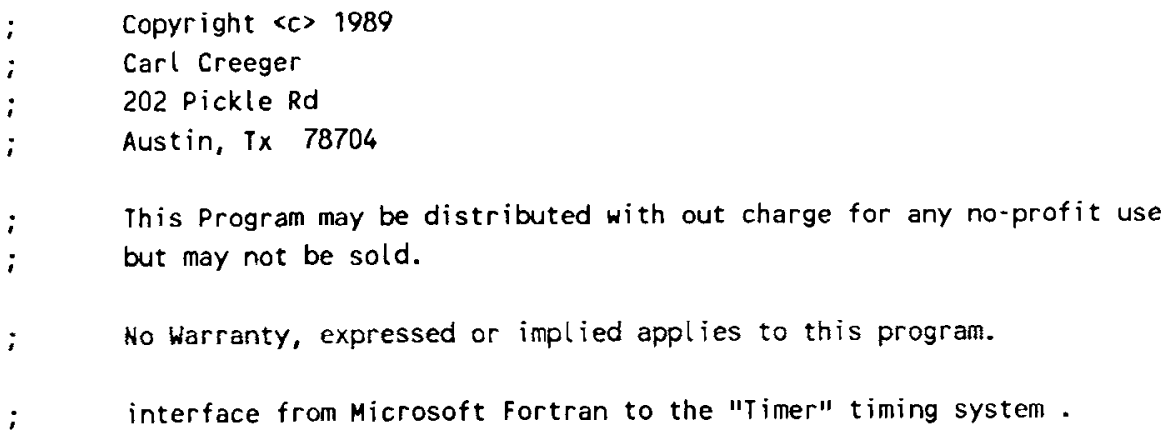




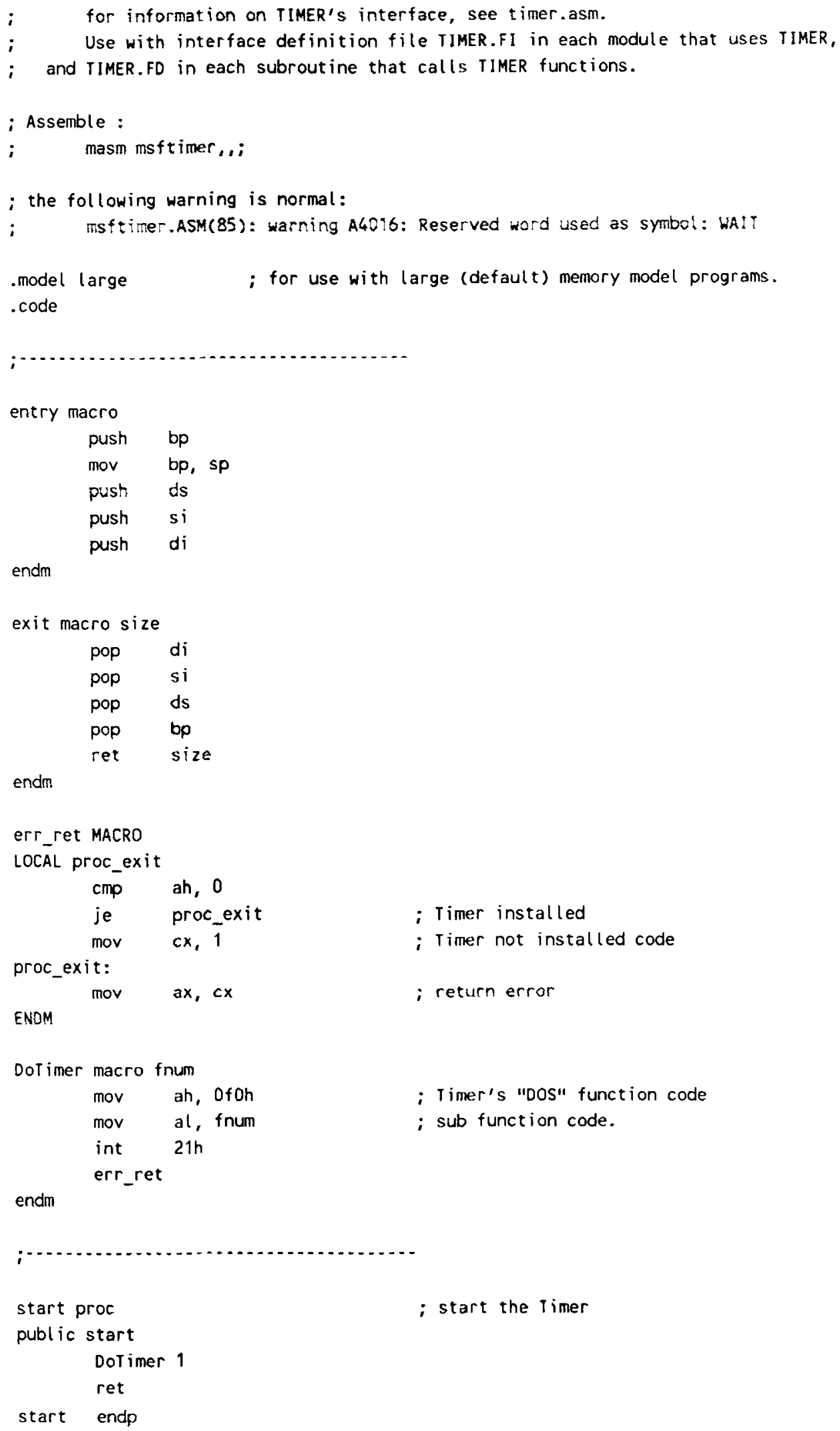


Listing 3 (Continued)

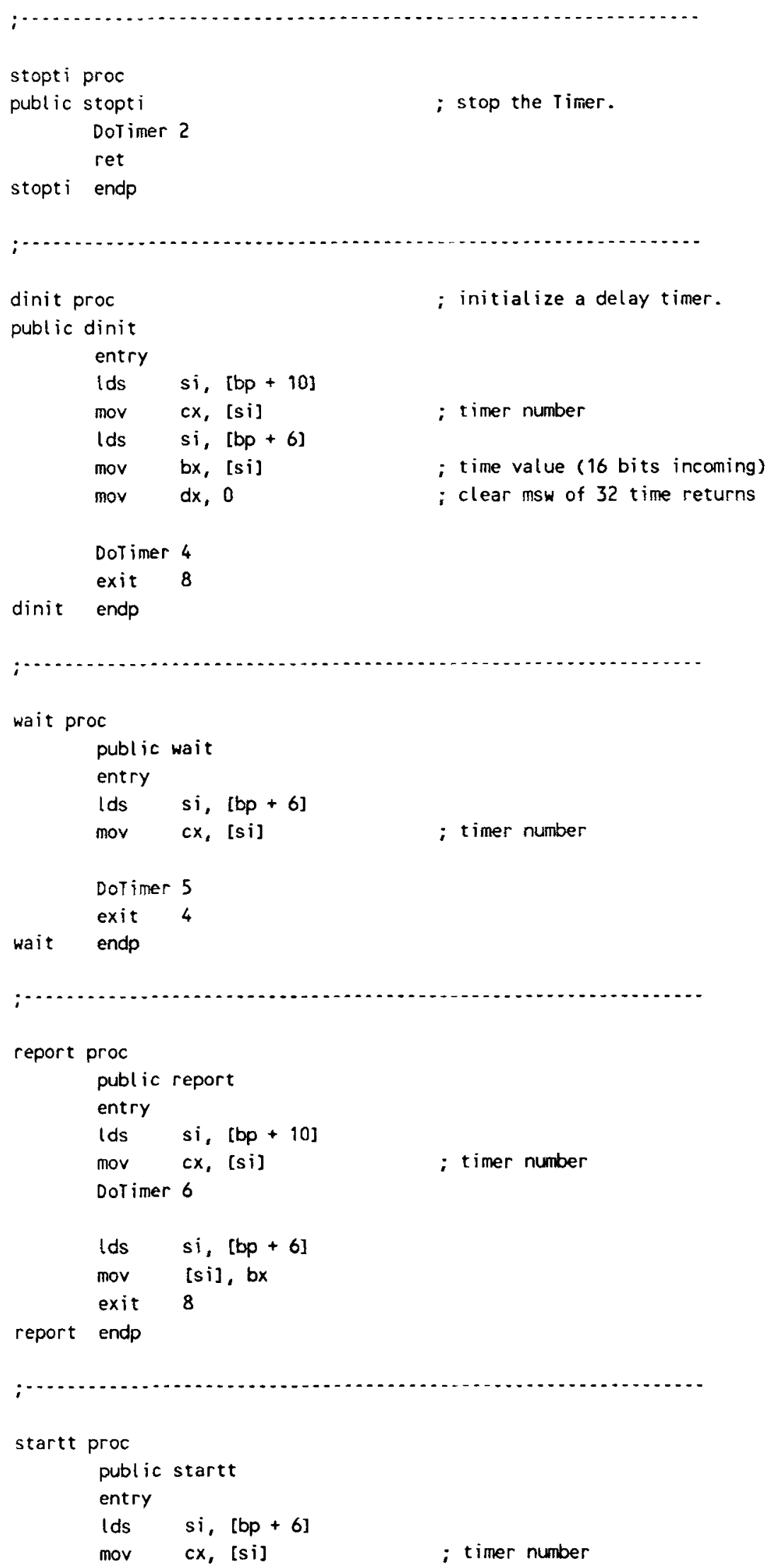




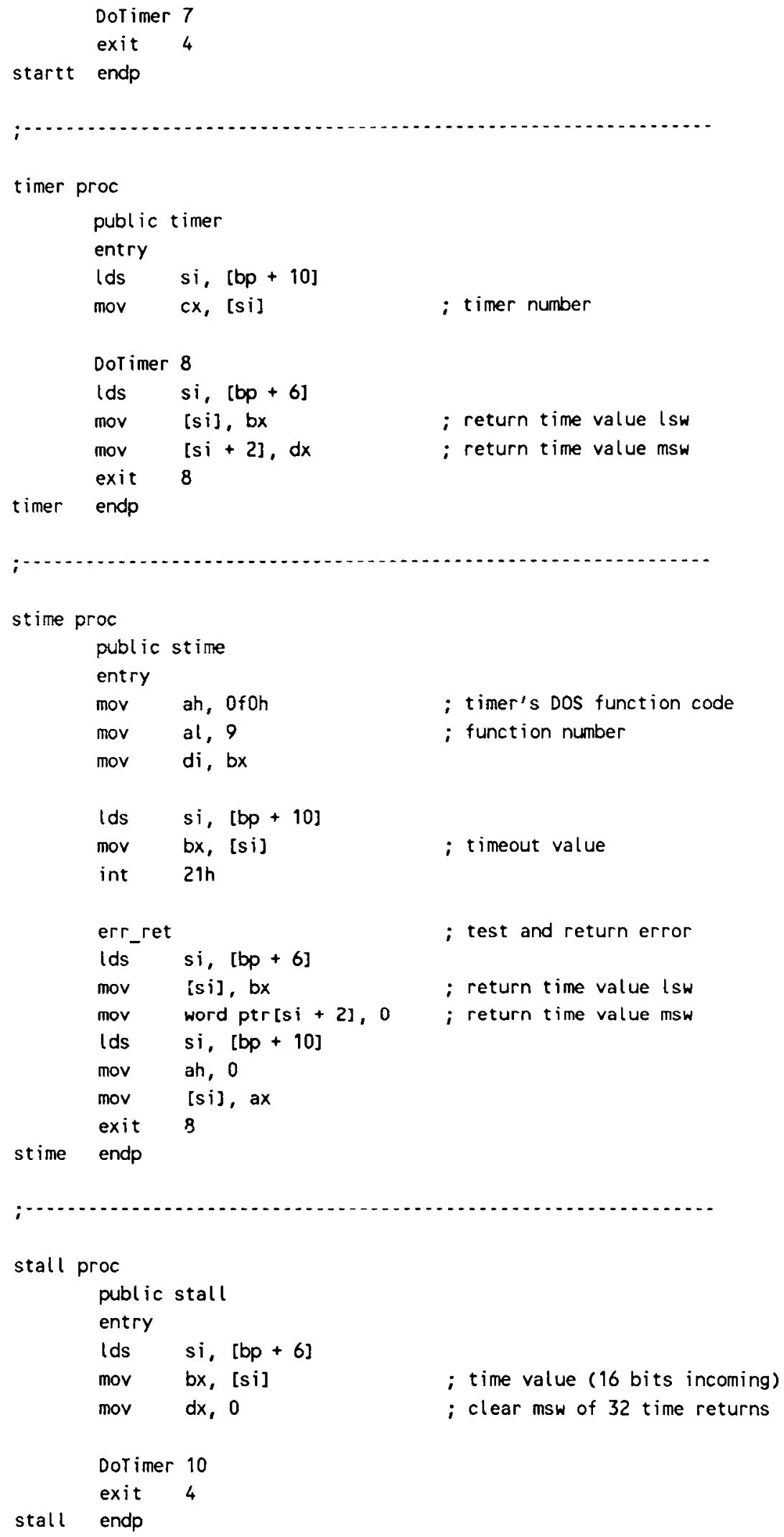


Listing 3 (Continued)

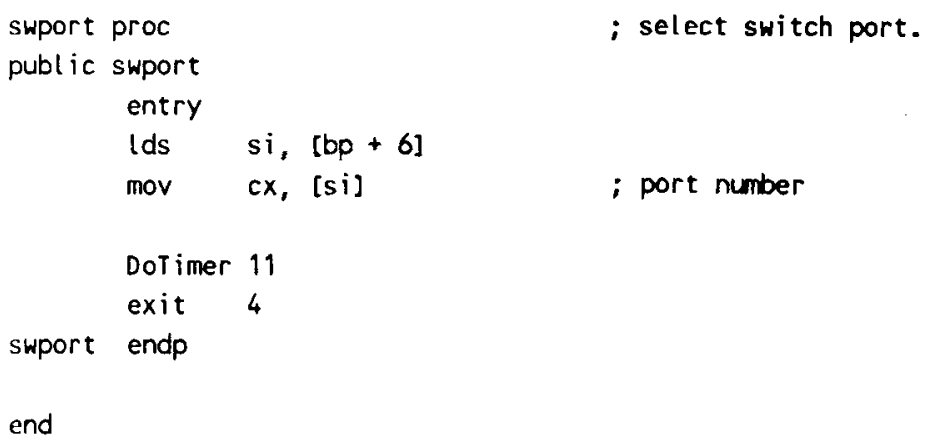

Listing 4: Microsoft QuickBASIC Language Interface for TIMER

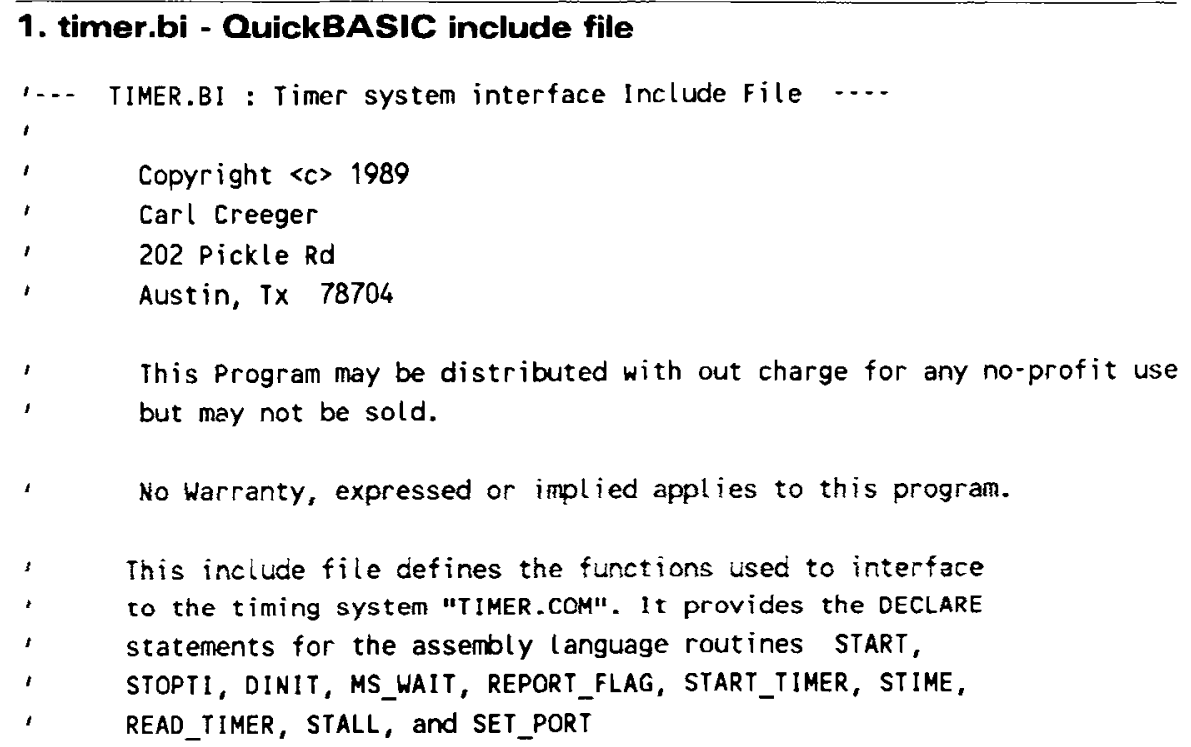


Listing 4 (Continued)

\section{2. qbtimer.asm - TIMER interface for Microsoft QuickBASIC}

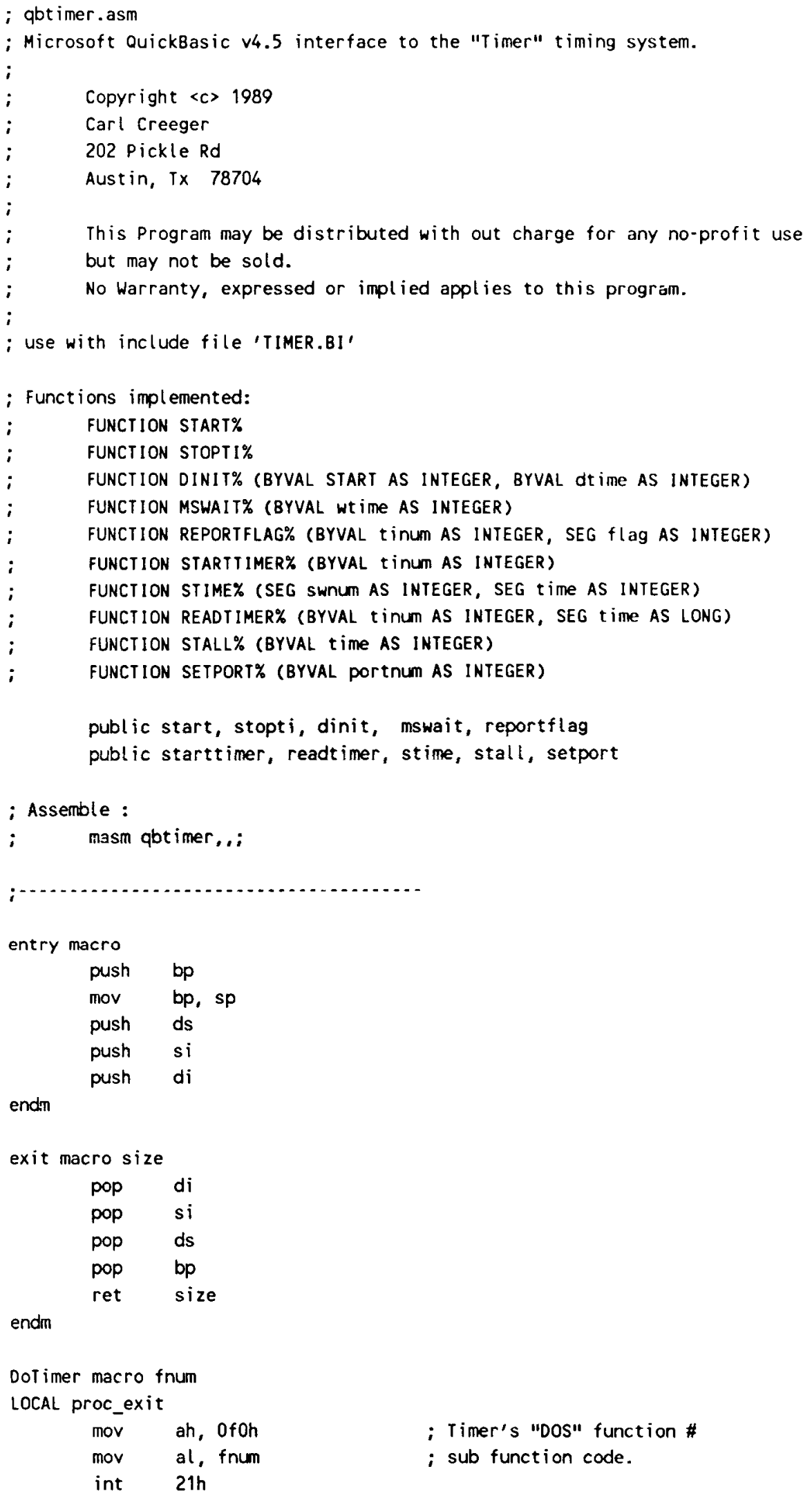


Listing 4 (Continued)

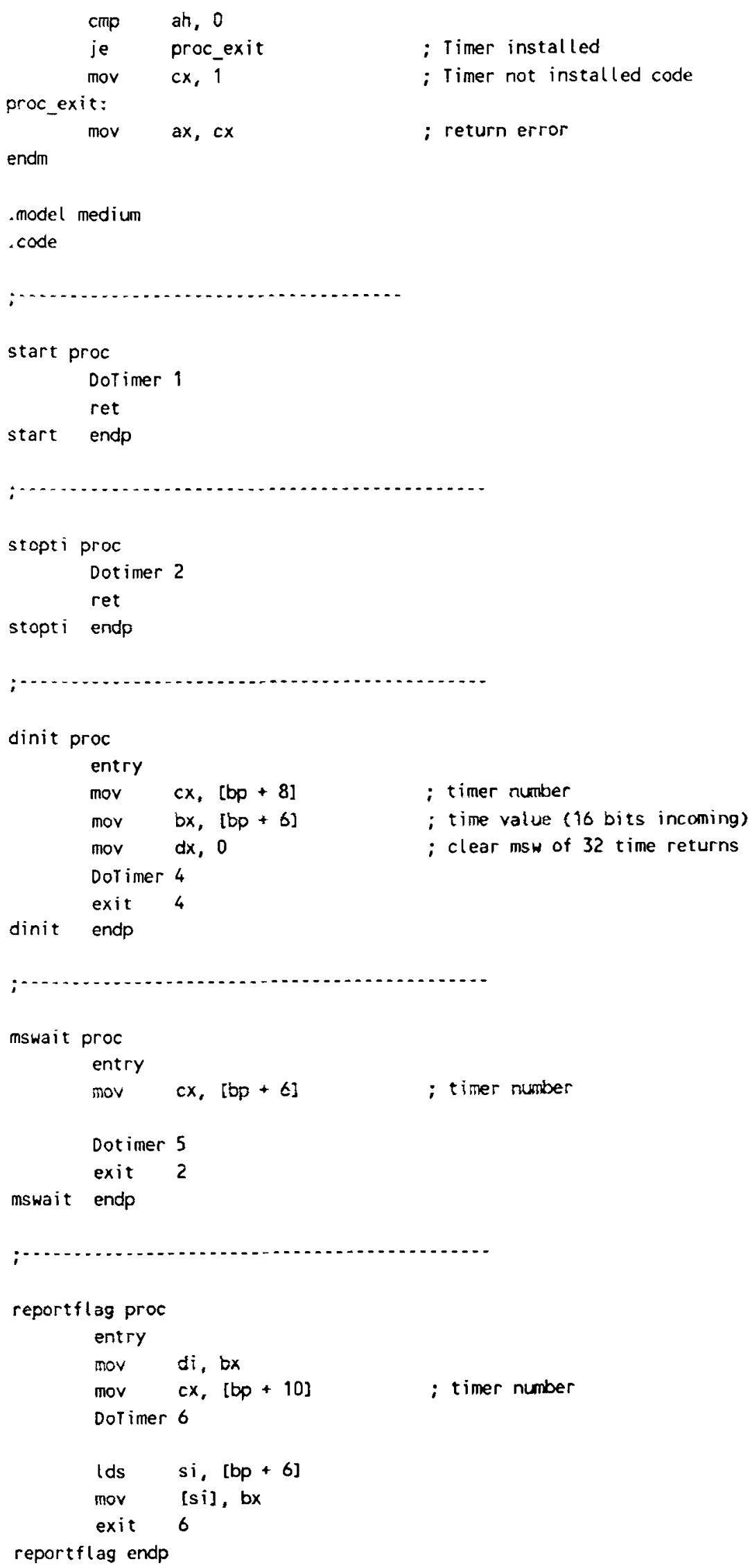




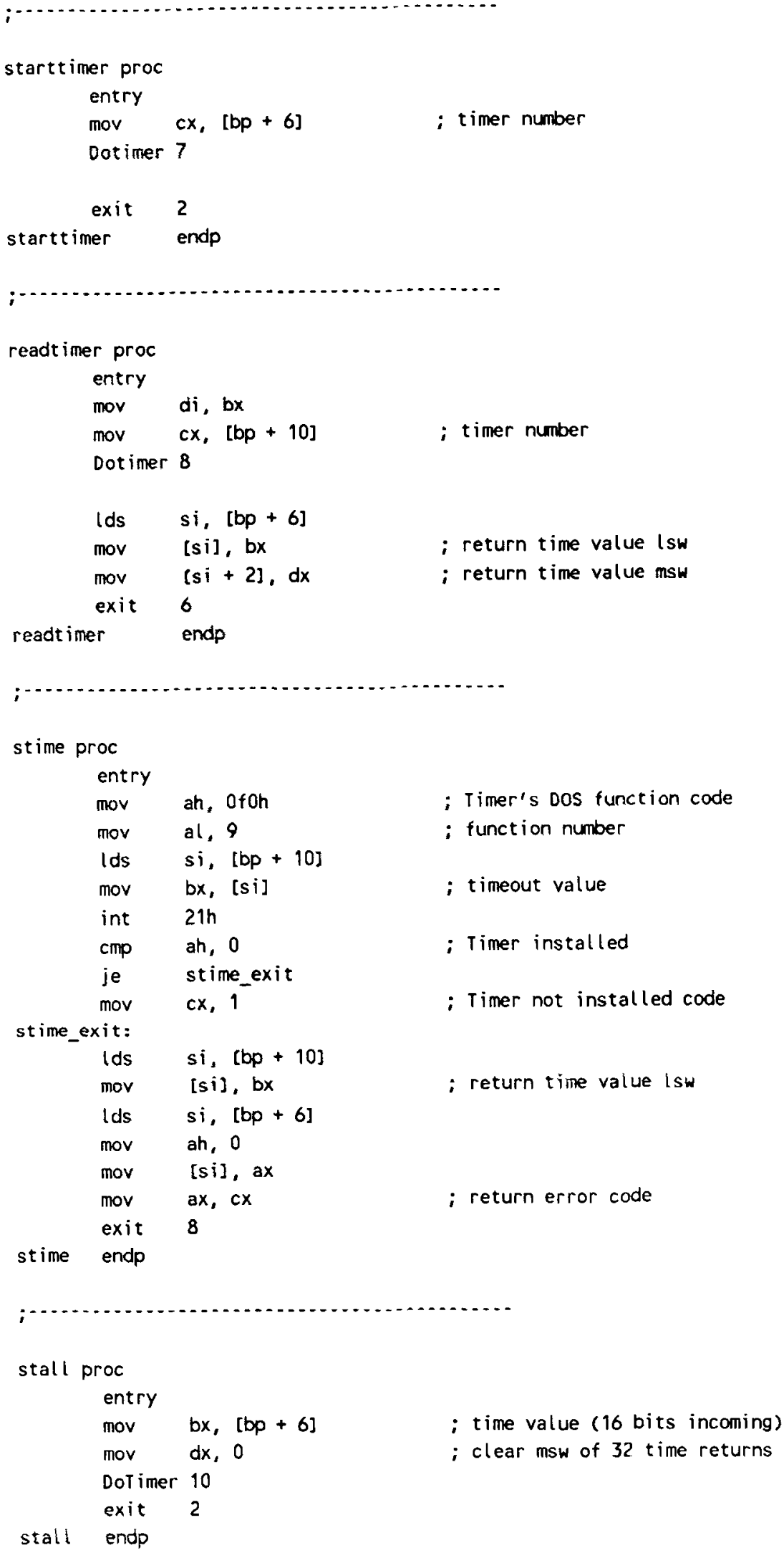


Listing 4 (Continued)

$\begin{aligned} & \text { setport proc } \\ & \text { entry } \\ & \text { mov } \\ & \text { Dotimer }\end{aligned}$
exit

Listing 5: TPTIMER.PAS: Turbo Pascal Interface to TIMER

1. IPIIMER.PAS

unit tptimer:

[This is an interface to the millisecond timing routine "timer"

See the file timer.doc for details.

Copyright <c> 1989
Carl Creeger
202 Pickle Rd
Austin, Tx 78704

This program may be distributed with out charge for any no-profit use but may not be sold.

No Warranty, expressed or implied applies to this program. 2

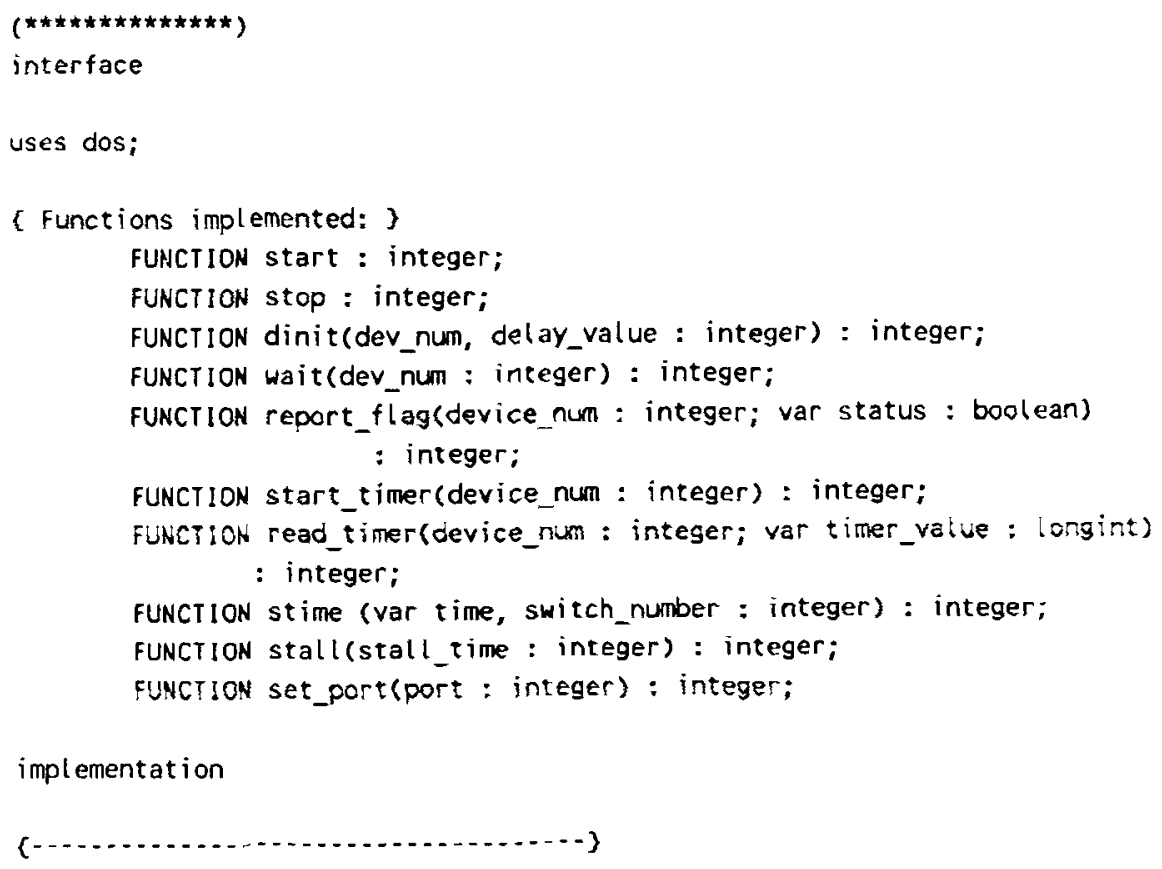




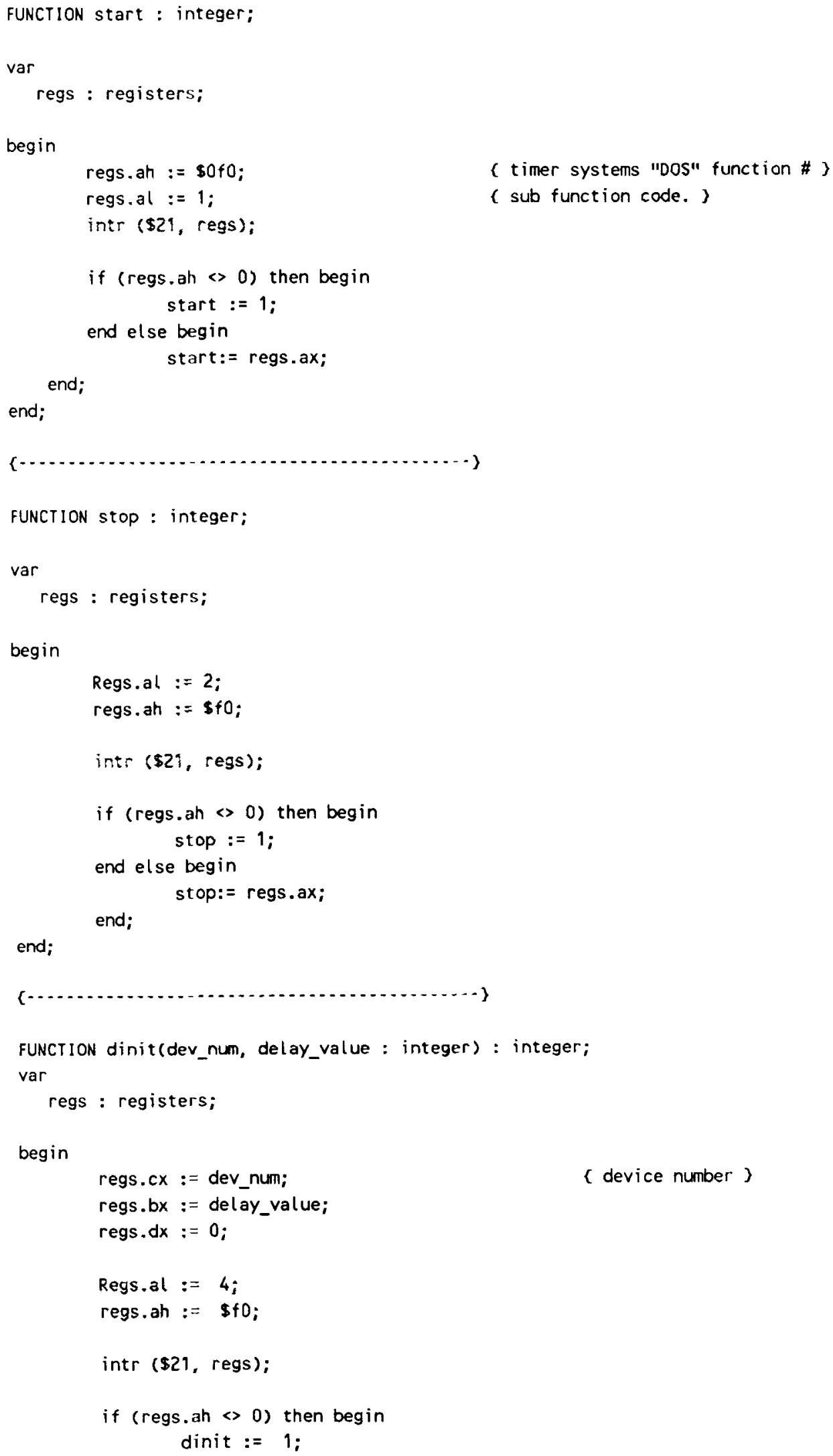


Listing 5 (Continued)

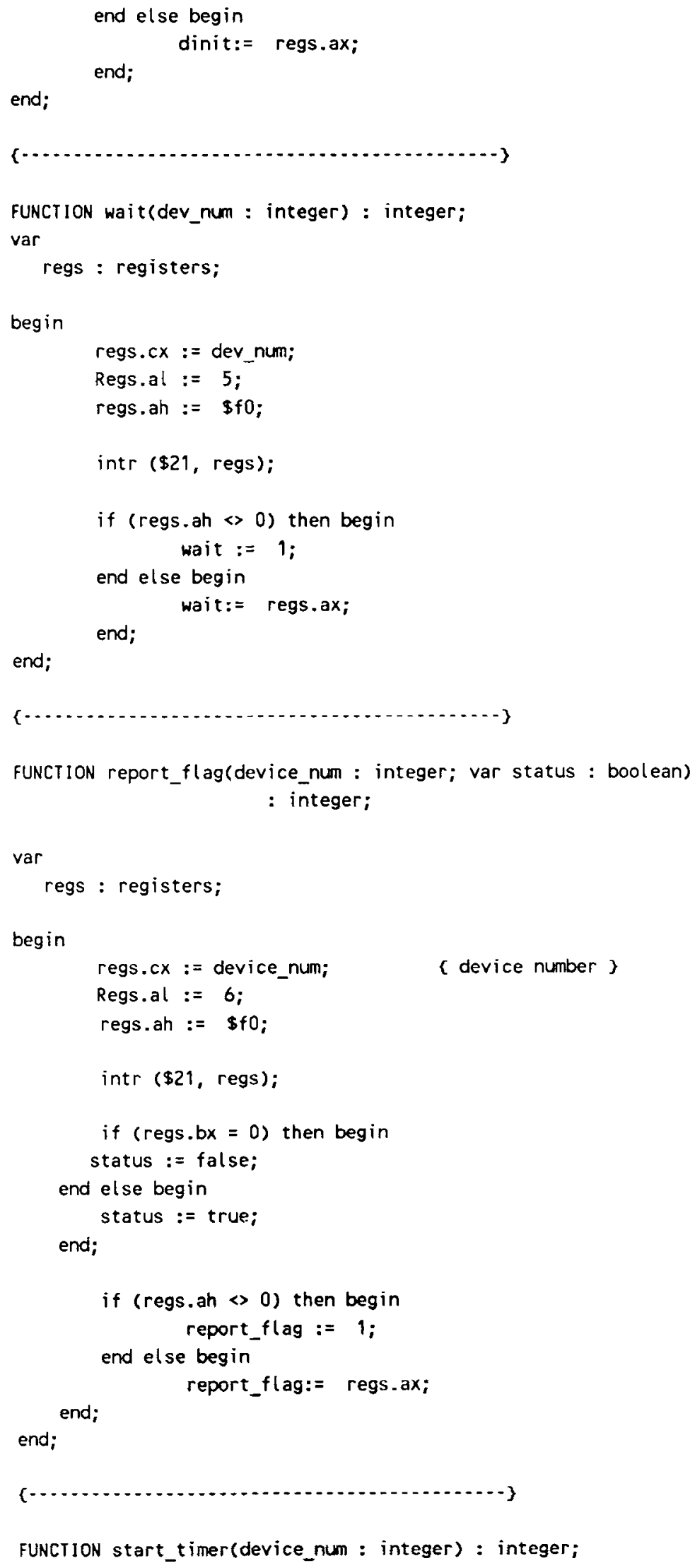

FUNCIION start_timer(device_num : integer) : integer; 


\section{Listing 5 (Continued)}

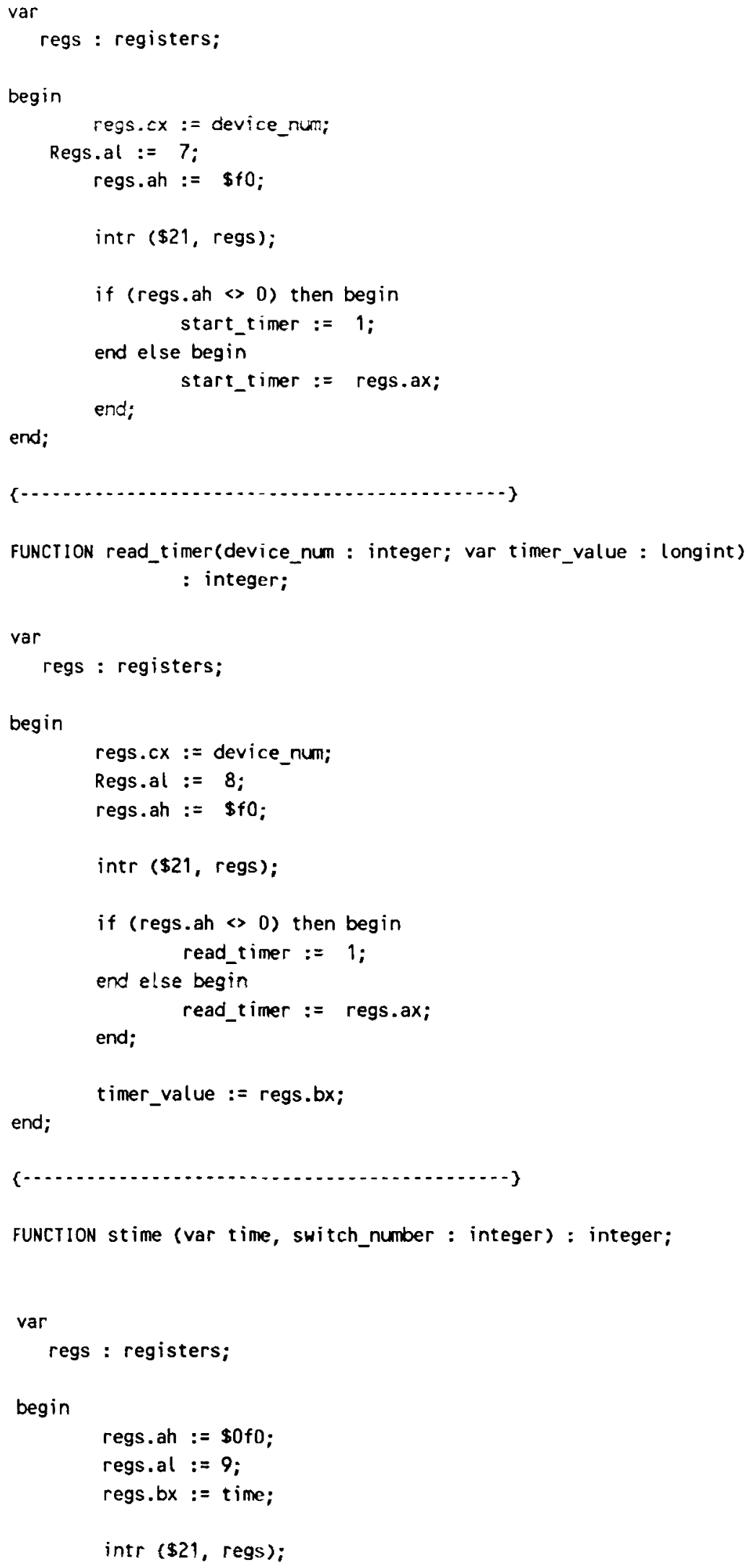




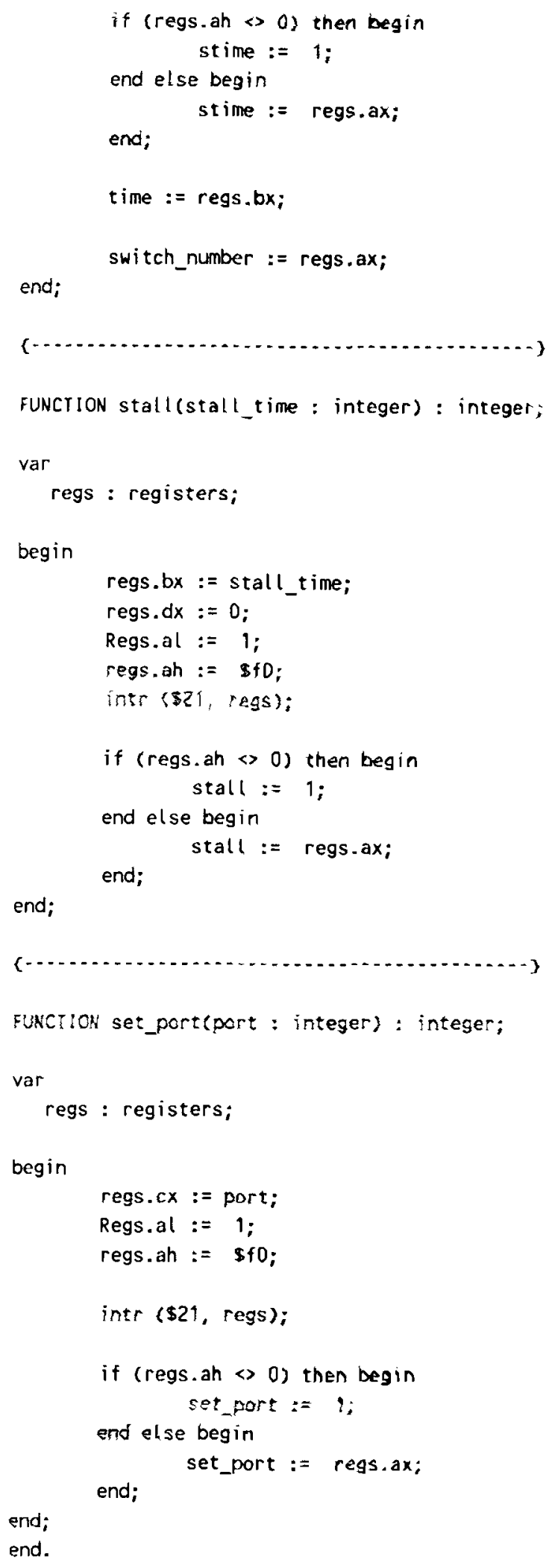


Listing 6: MicroMan.C: A Strategy for Measuring and Minimizing Timing Errors

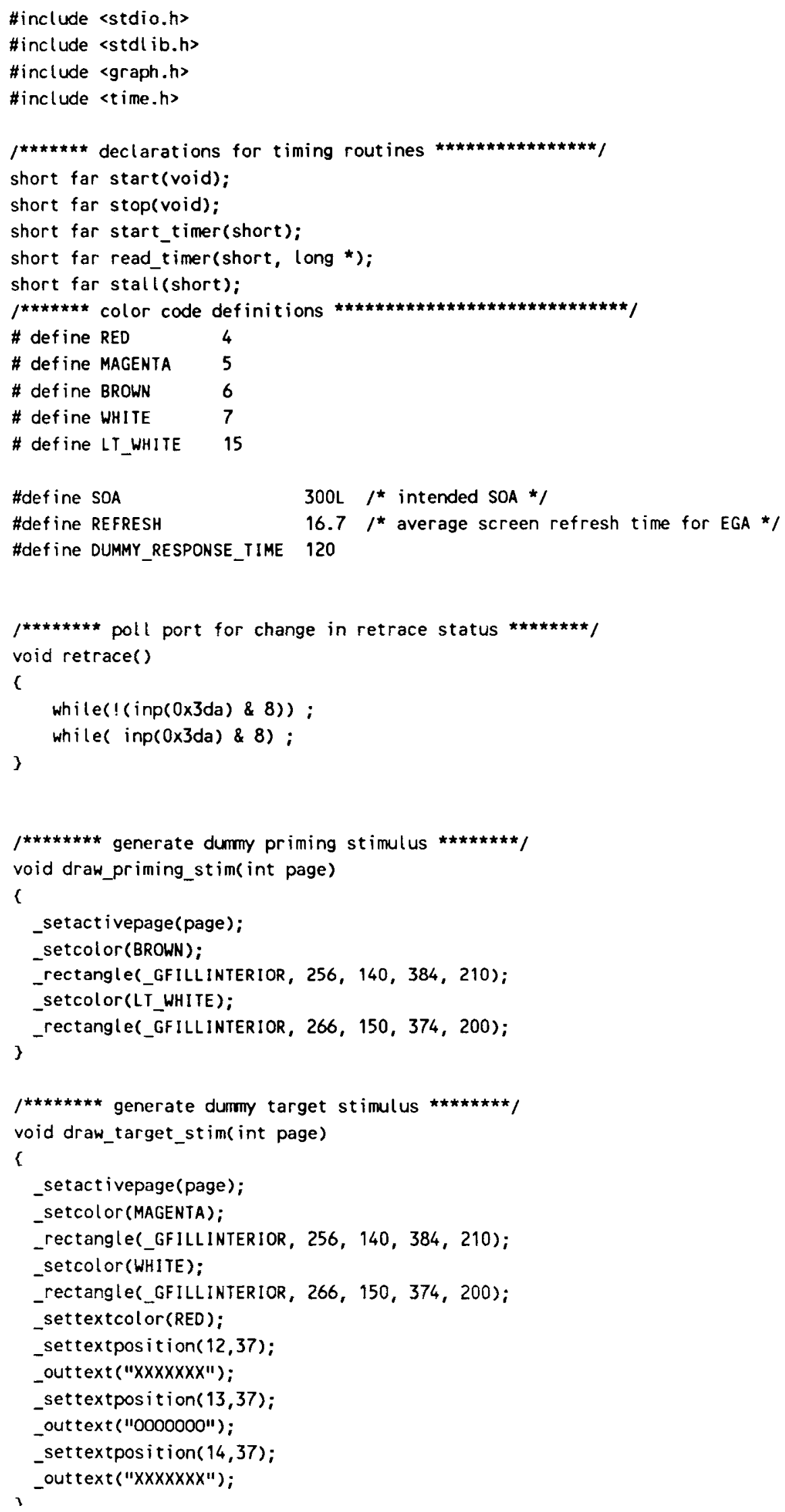


Listing 6 (Continued)

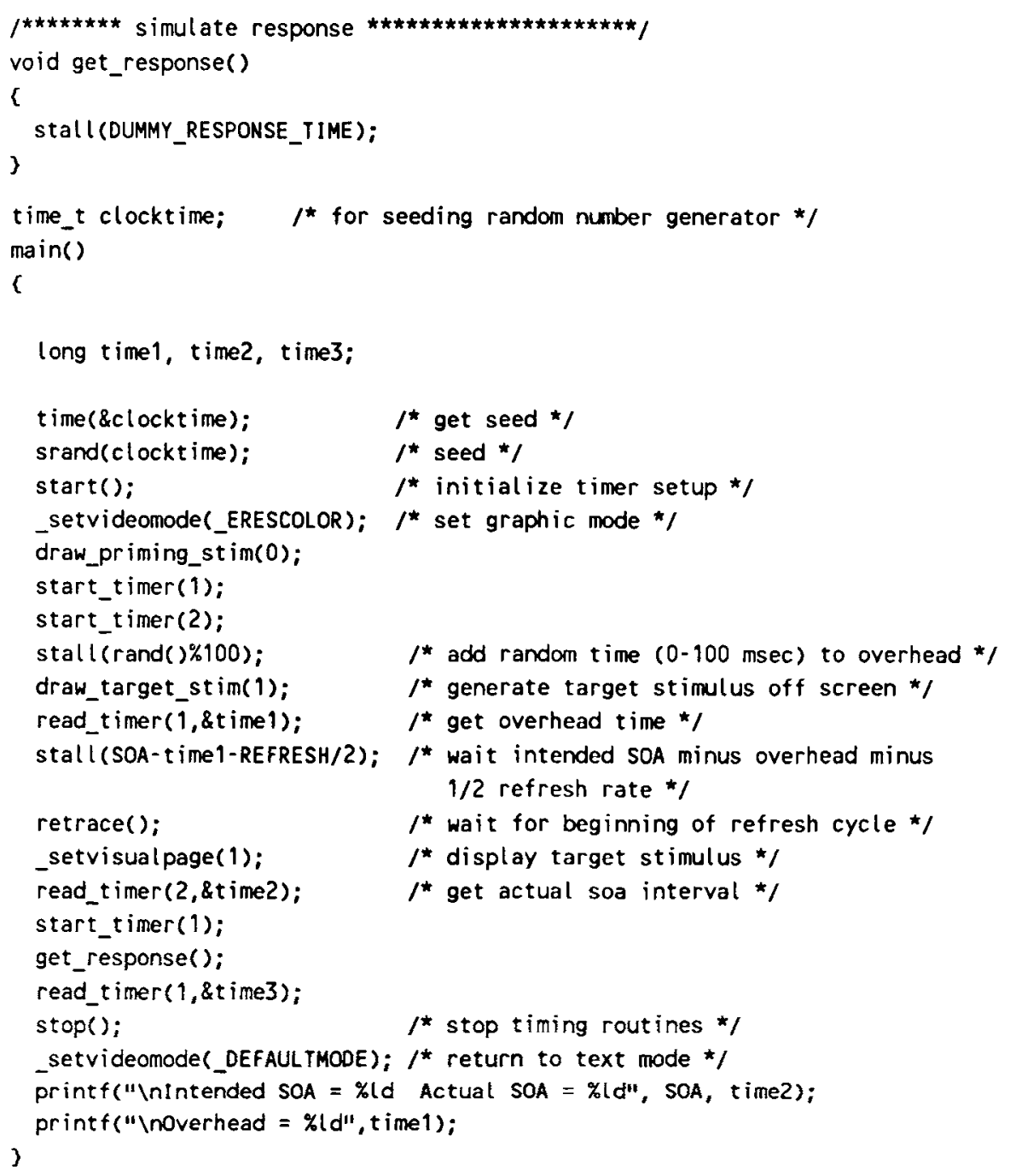

Listing 7: PsyBench.c: A Benchmark Program for Experimentally Relevant Tasks

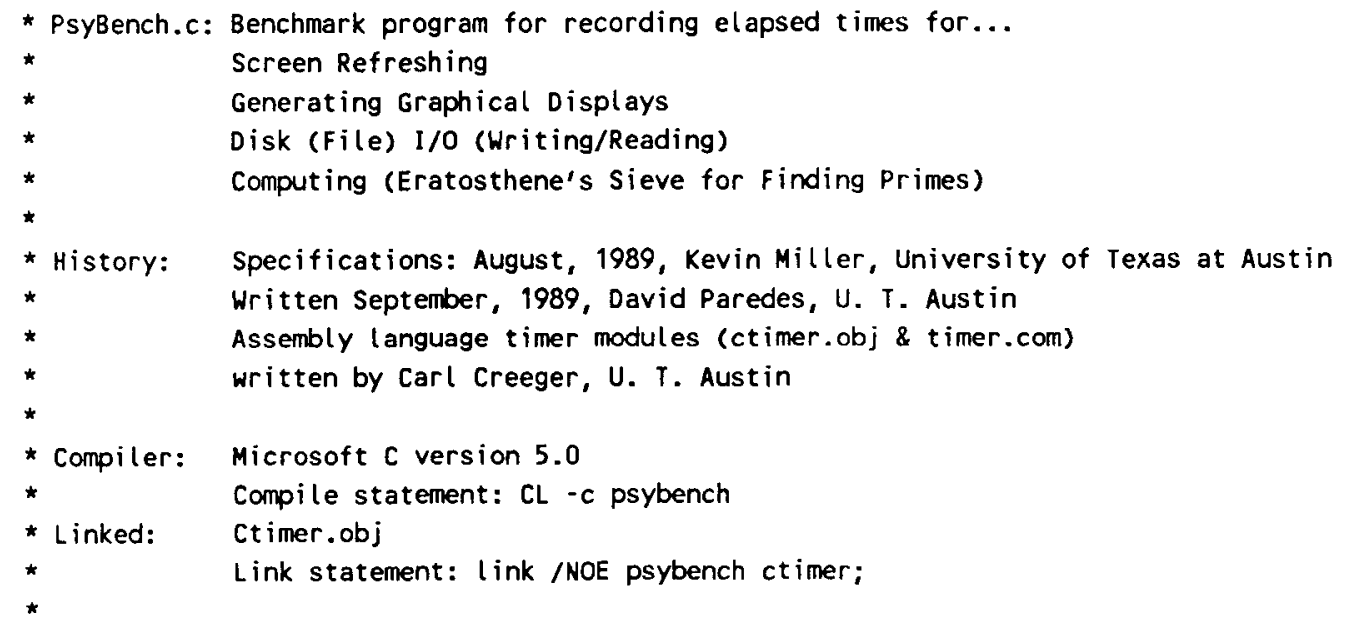

$\star$

\section{Screen Refreshing}

Generating Graphical Displays

Disk (File) I/O (Writing/Reading)

Computing (Eratosthene's Sieve for Finding Primes)

Specifications: August, 1989, Kevin Miller, University of Texas at Austin

Written September, 1989, David Paredes, U. T. Aust in

Assembly language timer modules (ctimer.obj \& timer.com)

written by Carl Creeger, U. T. Austin

Microsoft $C$ version 5.0

Compile statement: $\mathrm{CL}-\mathrm{c}$ psybench

ctimer.obj

Link statement: link /NOE psybench ctimer;

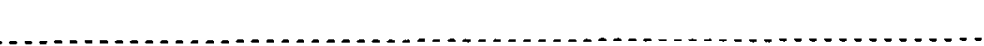


Listing 7 (Continued)

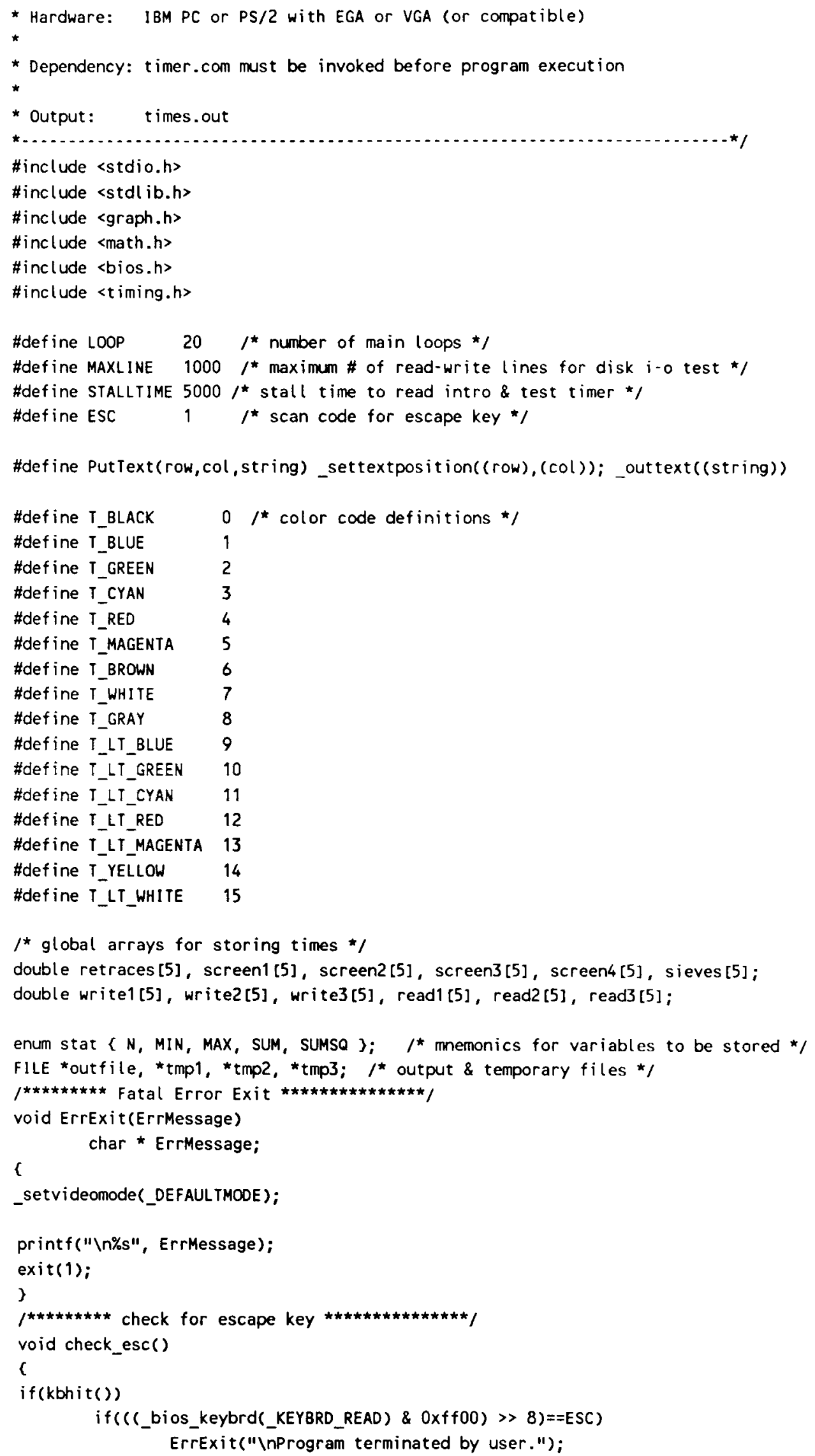




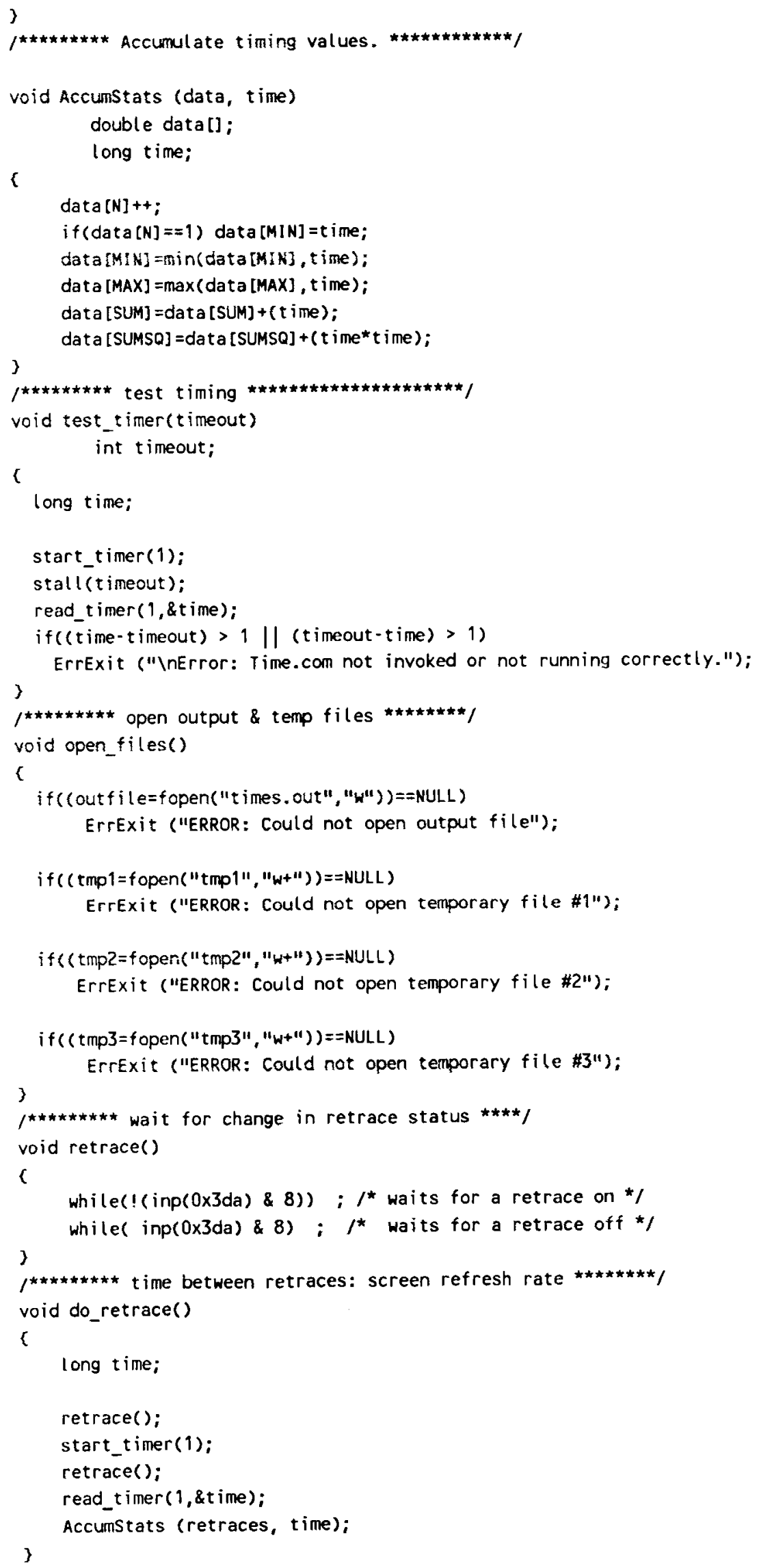




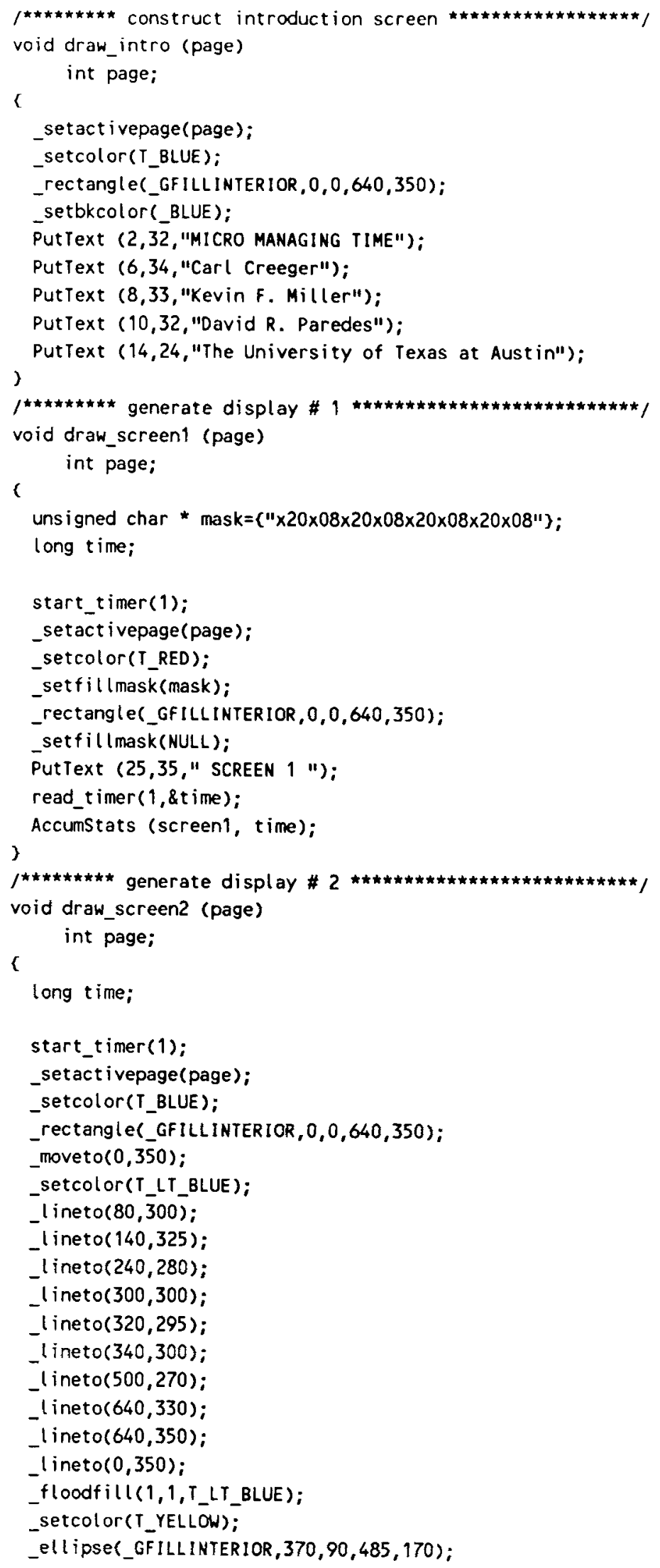




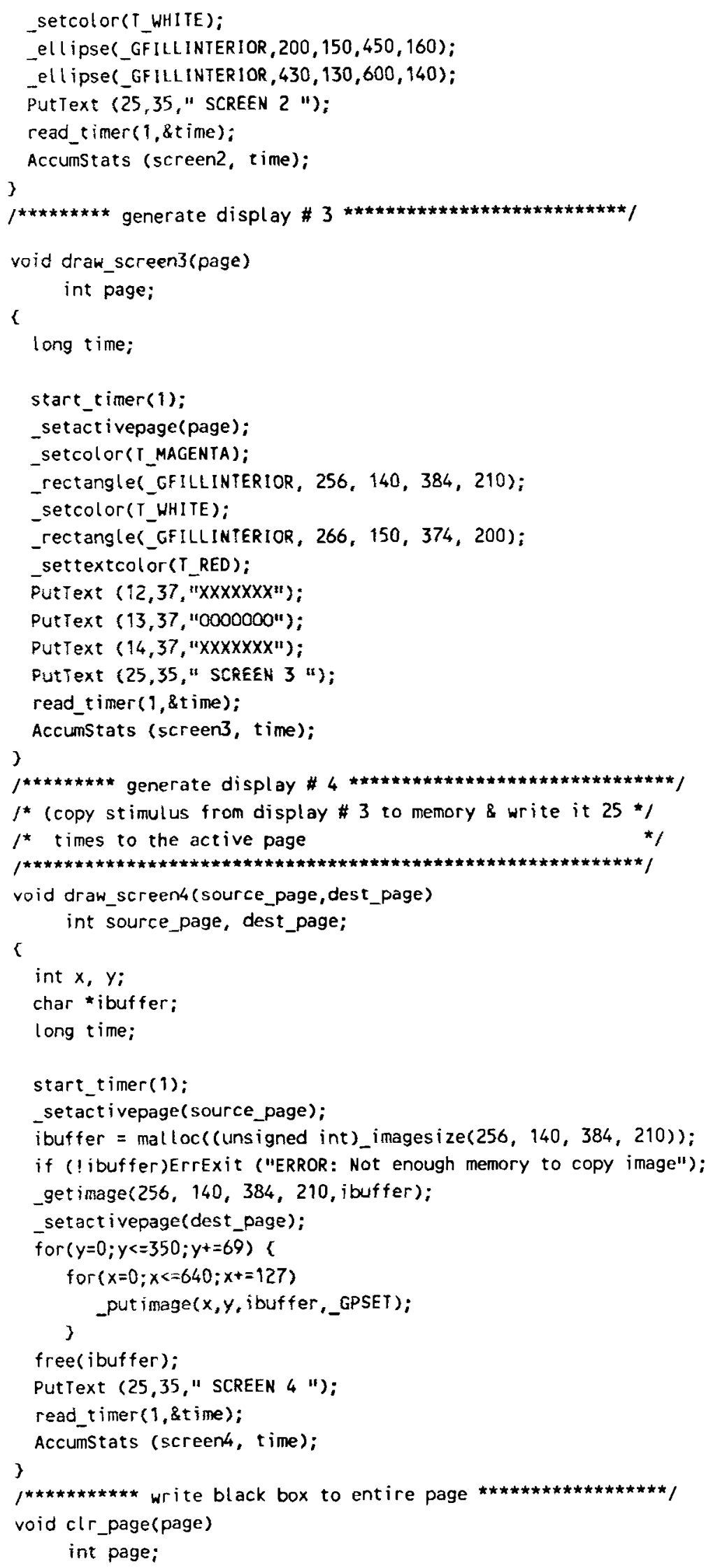




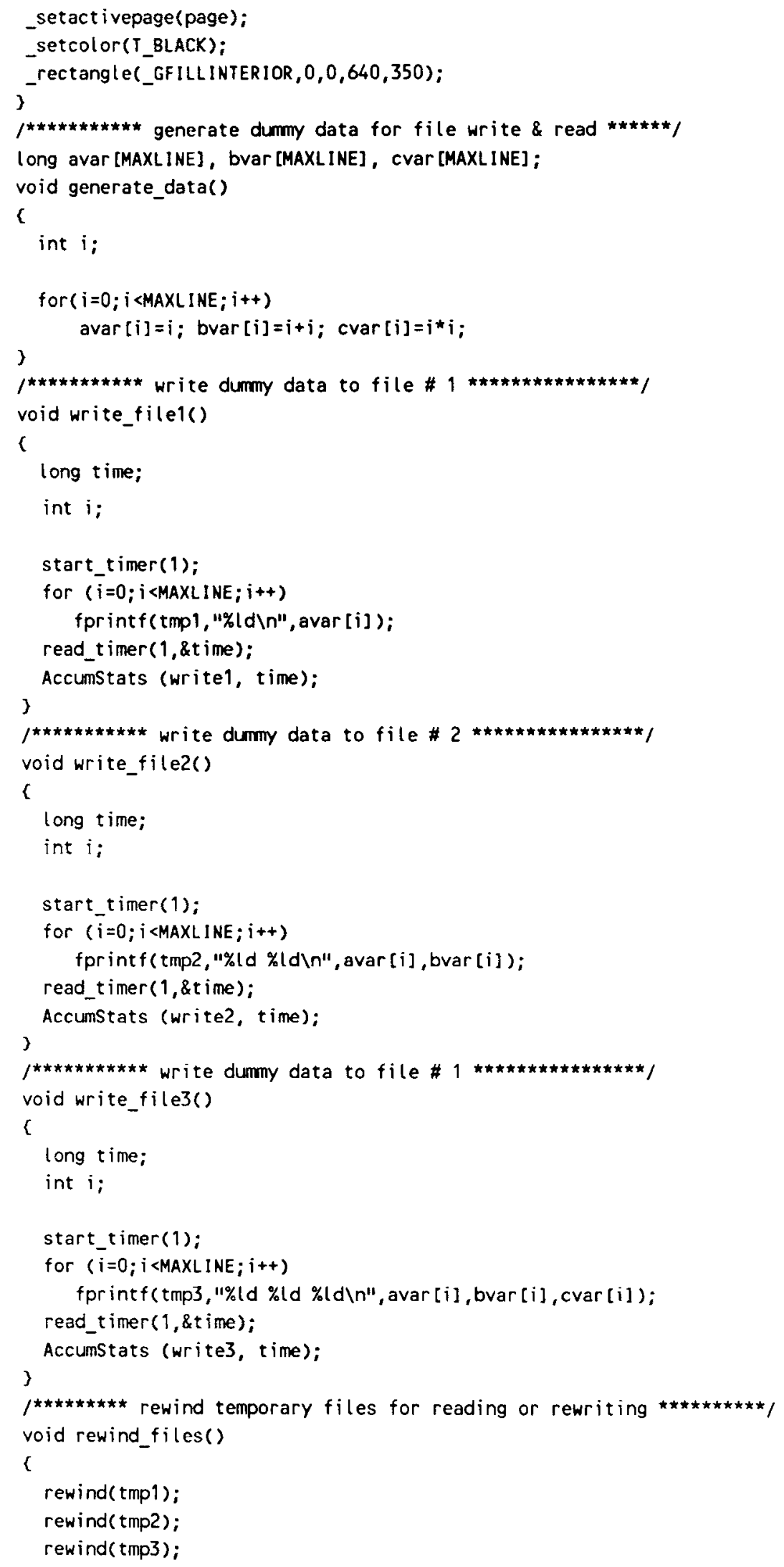


Listing 7 (Continued)

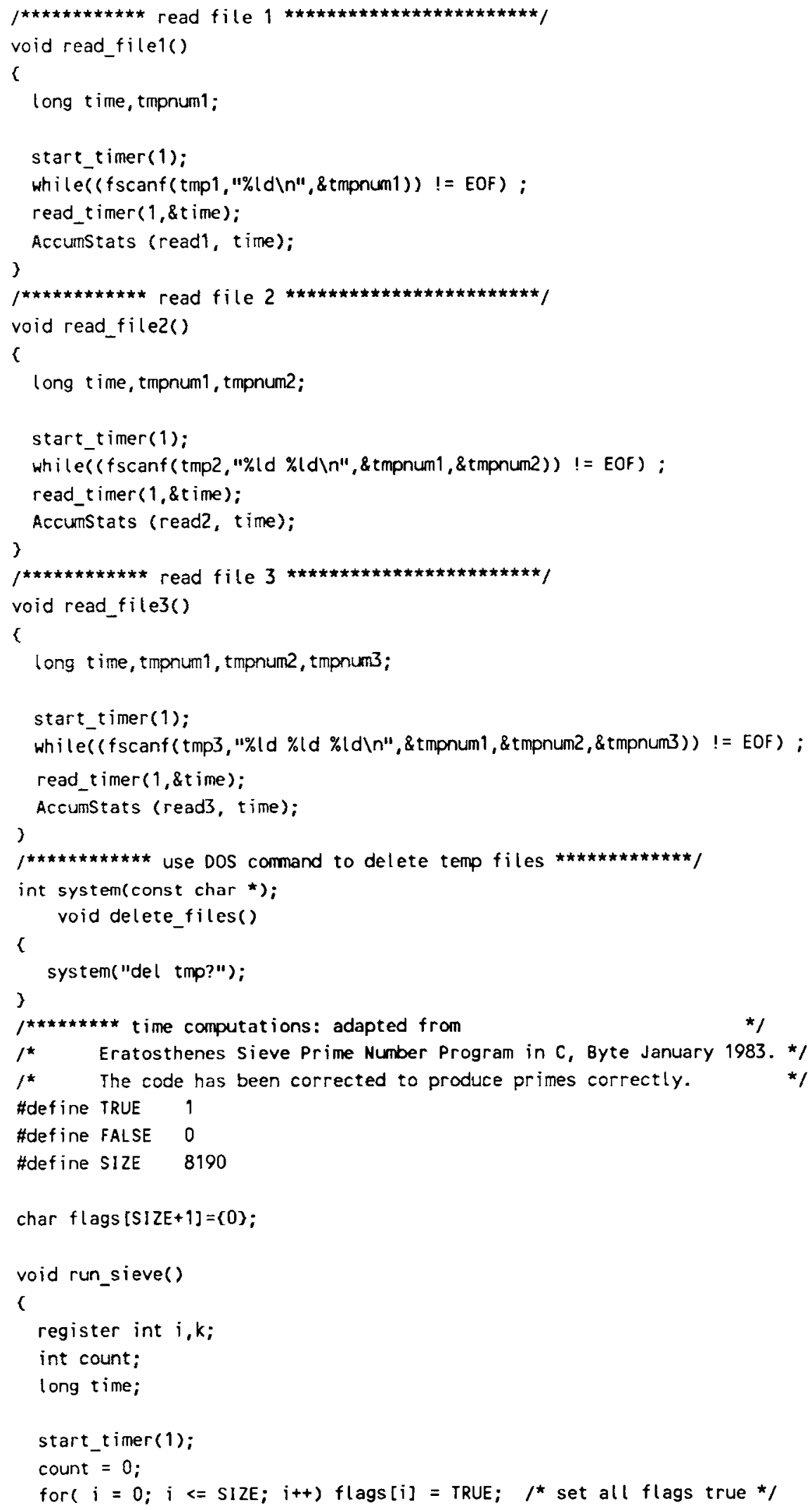


Listing 7 (Continued)

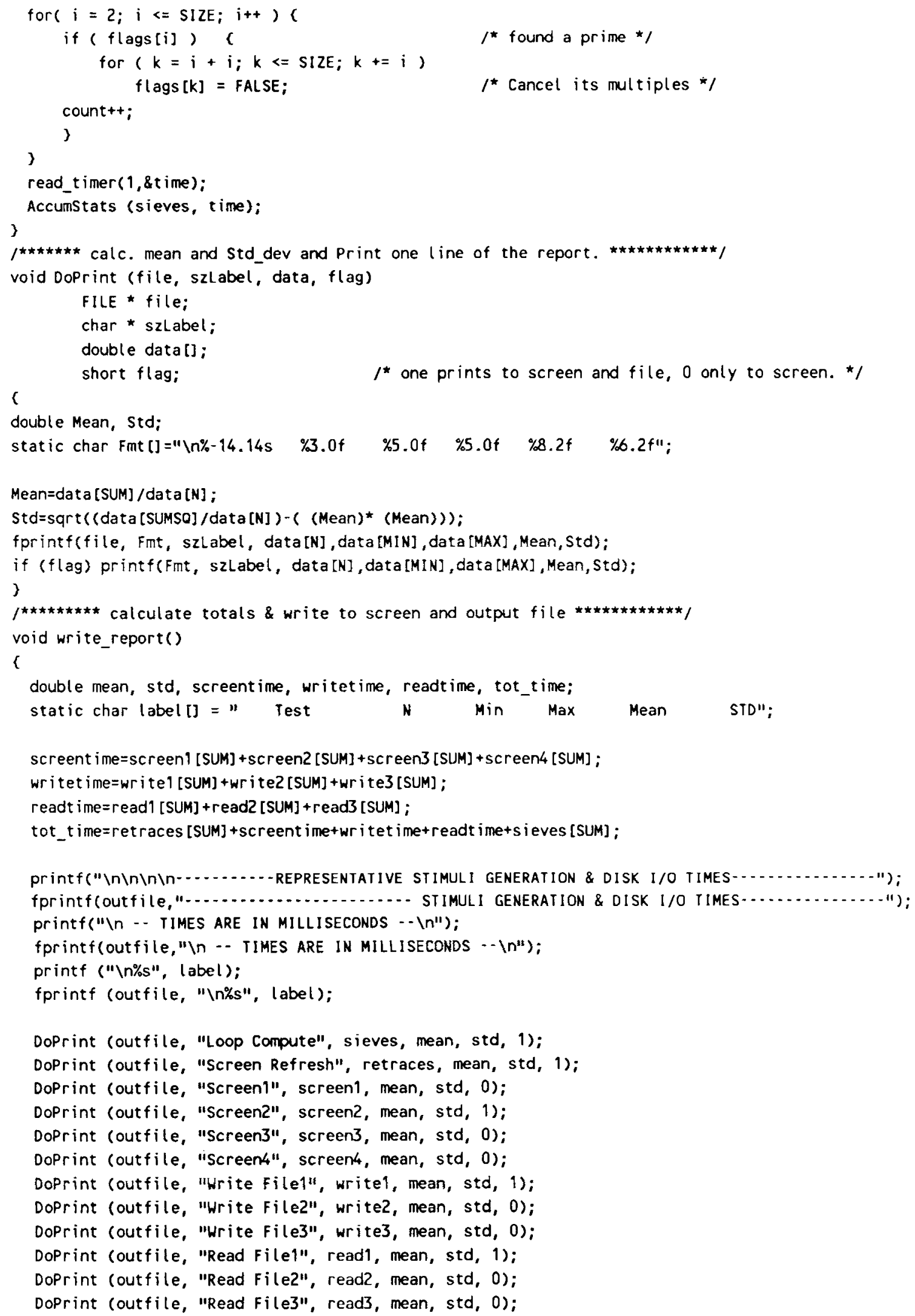




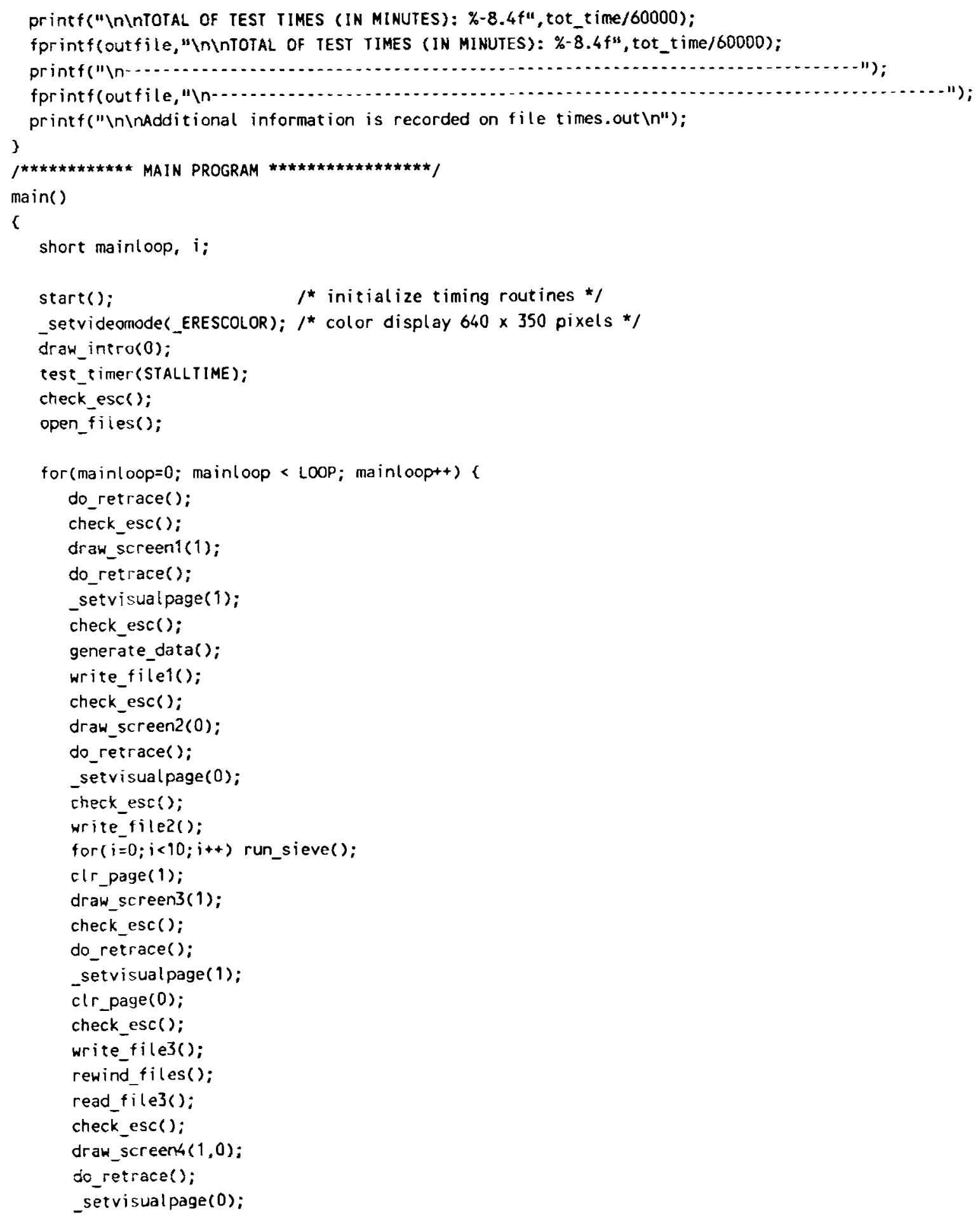




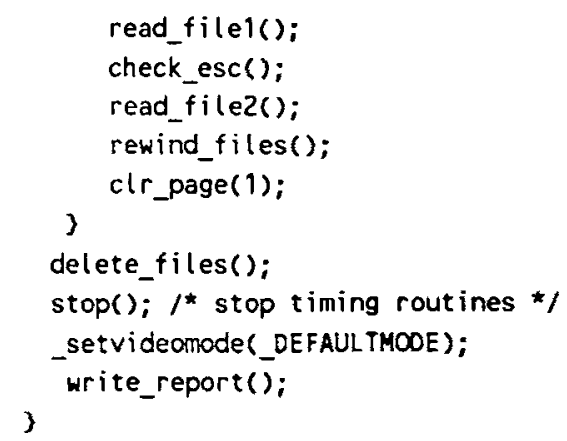

(Manuscript received October 5, 1989;

revision accepted for publication February 2, 1990.) 